\title{
Les Brunel père et fils : deux célèbres ingénieurs anglais « Made in France »
}

The Brunels, father and son: two famous British engineers "Made in France"

Jean-François Belhoste

\section{OpenEdition}

\section{Journals}

Édition électronique

URL : http://journals.openedition.org/dht/1369

DOI : $10.4000 /$ dht. 1369

ISSN : $1775-4194$

Éditeur :

Centre d'histoire des techniques et de l'environnement du Cnam (CDHTE-Cnam), Société des élèves du CDHTE-Cnam

Édition imprimée

Date de publication : 1 décembre 2010

Pagination : 131-152

ISBN : 978-2-9530779-5-7

ISSN : 0417-8726

Référence électronique

Jean-François Belhoste, «Les Brunel père et fils : deux célèbres ingénieurs

anglais « Made in France » », Documents pour l'histoire des techniques [En ligne], $19 \mid 2^{\mathrm{e}}$ semestre 2010

mis en ligne le 21 juin 2011, consulté le 08 septembre 2020. URL : http://journals.openedition.org/dht/

1369 ; DOI : https://doi.org/10.4000/dht.1369 


\title{
Les Brunel père et fils : deux célèbres ingénieurs anglais " Made in France "
}

\author{
Jean-François Belhoste \\ École pratique des Hautes Études (EPHE)
}

$\grave{A}^{\prime}$ I'automne 2002, la BBC organisa un grand référendum télévisé pour élire les Britanniques les plus célèbres, The greatest Briton. Arriva premier Winston Churchill, troisième Lady Diana et second Isambard Kingdom Brunel (1806-1859), assurément en France le moins connu des trois. II fut pourtant I'un des plus grands ingénieurs du XIXe siècle. Contemporain de Robert Stephenson (1803-1859), il fut certes longtemps moins honoré que lui, mais progressivement, ces dernières années, sa notoriété s'accrut au point qu'il est ainsi devenu en Angleterre premier sur la liste des héros de la révolution industrielle. Les raisons de ce choix sont diverses. Ce qui a été souligné, entre autres lors des fêtes commémoratives du bicentenaire de sa naissance en 2006 (sous le sigle Brunel 200), c'est un destin hors du commun pour cet ingénieur mort finalement assez jeune, à 53 ans, victime d'une certaine forme de surmenage'. Les Anglais admirent, en effet, son audace, qui le conduisit à faire des choix techniques pas forcément réalistes - en tout cas trop en avance sur leur temps et préjudiciables aux intérêts immédiats des capitalistes pour le compte desquels il travaillait, tout en manifestant une attitude d'apparence quelque peu désinvolte qui n'était pas partagée par la plupart de ses concurrents, en particulier Robert Stephenson. La diversité de ses interventions permet d'expliquer aussi ce grand succès posthume. Alors que Robert Stephenson, comme déjà son père George (1781-1848), est d'abord reconnu pour ses réalisations ferroviaires, Isambard Kingdom Brunel l'est certes aussi pour la construction du Great Western Railway (ligne Londres-Bristol construite entre 1835 et 1841) et de ses lignes satellites (vers Oxford, le South-Devon, la Cornouaille et le sud du Pays de Galles) avec ses ponts, ses tunnels, ses gares, la ville nouvelle de Swindon créée à partir de 1841 pour accueillir la construction et l'entretien du matériel, mais aussi ses

1 Andrew Kelly, Melanie Kelly éd., Brunel. In love with the impossible. A celebration of the life, work and legacy of Isambard Kingdom Brunel, Bristol, Bristol Cultural Development Partnership, 2006. travaux portuaires à commencer par ceux réalisés pour Bristol à partir de 1833 , et surtout la conception et la réalisation entre 1836 et 1859 de trois remarquables navires transocéaniques (Great Western, Great Britain et Great Eastern). Comme Robert Stephenson, Isambard Kingdom Brunel avait aussi un père ingénieur, Marc Isambard (1769-1849), célèbre entre autres pour la construction entre 1825 et 1843 du premier tunnel réalisé à Londres sous la Tamise, chantier, du reste, sur lequel débuta son jeune fils. La place éminente occupée par les Brunel père et fils, tient encore à la conservation d'un important patrimoine les concernant, tant écrit et figuré que matériel. On dispose, en effet, non seulement d'une abondante documentation sur leurs travaux dans les archives publiques², mais aussi de papiers personnels journaux de travail, correspondances, livres comptables déposés à I'Université de Bristol $^{3}$ et à I'Institution of Civil Engineers à Londres ${ }^{4}$. En plus, subsistent bon nombre de leurs ouvrages qui profitant de l'intérêt porté depuis une quarantaine d'années au patrimoine industriel et technique, ont fait l'objet d'études et de mesures de protection et de mise en valeur : Block Mills à Portsmouth achevé par Marc Isambard en 1805 ; le tunnel sous la Tamise emprunté aujourd'hui par le métro londonien (East London) ; I'ancien bâtiment des machines du dit tunnel (Brunel Engine House) devenu Brunel Museum à Rotherhithe (sud-est de Londres) ; les gares terminales du GWR à Londres (Paddington Station) et à Bristol (Temple Meads) et de nombreux ouvrages d'art le long de la ligne (viaduc et tunnel de Twerton, tunnel de Box, viaduc de Warncliffe, pont de Maidenhead...) ; les ateliers et cités

2 Entre autres aux National Archives, British Rail Archive pour les lignes de chemins de fer et Bristol City Archives pour les travaux dans le port de Bristol.

3 « Brunel Collection », papiers remis en 1950 par la petite fille d'Isambard K., Celia Brunel Noble en 1950. Nick Lee, "The Brunel Collection, Bristol University Librairy », dans A. Kelly, M. Kelly éd., Brunel. In love..., op. cit., p. 192-203.

4 Concernant principalement Marc Isambard : journaux de travail (diaries), mais aussi papiers du chantier du tunnel sous la Tamise. 


\section{Les Brunel père et fils : deux célèbres ingénieurs anglais « Made in France »}

ouvrières de la ville ferroviaire de Swindon où a été créé le Musée du Great Western Railway - STEAM ; le bâtiment des machines du chemin de fer atmosphérique du South Devon à Torquay ; les écluses du Floating Harbor de Bristol; le navire Great Britain restauré entre 1998 et 2005 et visitable depuis dans ce même port; deux ponts remarquables, enfin, celui suspendu de Clifton au dessus des gorges de l'Avon à Bristol (commencé en 1831 et terminé seulement en 1864) et le Royal Albert Bridge de Saltash, franchissant l'estuaire du Tamar entre le Devon et la Cornouaille (construit entre 1855 et 1859). Cette abondante matière documentaire a donné lieu, bien entendu, à un grand nombre de biographies, les premières remontant au XIXe siècle ${ }^{5}$, les autres plus récentes, entre autres, celles de Célia Brunel Noble (1938) ${ }^{6}$, de L.T.C. Rolt (1957) ${ }^{7}$, de Paul Clements $(1970)^{8}$, d'Angus Buchanan (2001) ${ }^{9}$, I'un des pères de I'archéologie industrielle en Grande-Bretagne, et de Steven Brindle $(2005)^{10}$. S'y ajoutent les travaux consacrés spécifiquement à leurs réalisations: Block Mills de Portsmouth ${ }^{11}$, ouvrages du Great Western Railway ${ }^{12}$, gare de Paddington ${ }^{13}$, ville ferroviaire de Swindon ${ }^{14}$, ligne de chemin de fer du Sud du Pays de Galles (South Wales) ${ }^{15}$, navires transocéaniques ${ }^{16}$... Le projet initié en 1999 par

5 Richard Beamish, Memoir of Sir Marc Isambard Brunel, Londres, 1862. Isambard Brunel fils, The life of Isambard Kingdom Brunel, civil engineer, Londres, 1870.

6 Celia Brunel Noble, The Brunel: Father and Son, Londres, Cobden-Sanderson, 1938.

7 L.T.C. Rolt, Isambard Kingdom Brunel, Londres, Longmans Green, 1957.

8 Paul Clements, Marc Isambard Brunel, Londres, Longmans Green, 1970.

9 Angus Buchanan, Brunel. The Life and Times of Isambard Kingdom Brunel, Londres, Hambledon Continuum, 2001. Les premiers travaux d'A. Buchanan sur I. K. Brunel à Bristol remontent aux années 1970.

10 Steven Brindle, The man who built the world, Londres, Weidenfeld and Nicholson, 2005. Citons encore sans épuiser le sujet: John Pudney, Brunel and his word, Londres, Book Club Associates, 1974, Adrian Vaughan, Isambard Kingdom Brunel. Engisneering Knight-Errant, Londres, John Murray Publishers, 1991. 11 Jonathan Coad, The Porthsmouth Block Mills. Bentham, Brunel, and the start of the Royal Navy's Industrial Revolution, Swindon, English Heritage, 2005.

12 Steven Brindle, "The Great Western Railway », dans A. Kelly, M. Kelly éd., Brunel. In love..., op. cit., p. 132-155.

13 Steven Brindle, Paddington Station. Its history and architecture, Swindon, English Heritage, 2004.

14 John Catell, Keith Falconer, Swindon. The legacy of a Railway Town, Swindon, English Heritage, 1995.

15 Stephen Jones, Brunel in South Wales, vol. 1, In Trevithick's tracks, Stroud, Tempus, 2005.

16 John Dugan, The Great Iron Ship, Harper and Brothers, New- les services officiels du patrimoine - English Heritage - de faire inscrire au patrimoine mondial de l'Unesco - World Heritage List - la ligne Londres-Bristol, comme étant la mieux conservée, du fait de sa qualité, des premières lignes britanniques, a donné une forte impulsion à ces études. English Heritage a, du reste, installé ses bureaux dans les anciens bureaux du GWR à Swindon.

Dans tous ces livres, I'origine française des Brunel n'est généralement évoquée que de façon rapide. Et pourtant Marc Isambard, le père, était né en Normandie, avait fait ses études à Rouen et ne s'était installé en Angleterre qu'à 32 ans, en 1799. Et si Isambard Kingdom était bien né lui à Portsmouth, d'une mère anglaise, il avait fait une partie de ses études à Paris. Ces faits, les Français semblent les avoir oubliés, et les Brunel leur sont aujourd'hui devenus pratiquement inconnus, les Stephenson étant depuis longtemps pour eux les seuls à symboliser le rôle de l'Angleterre dans la révolution industrielle ${ }^{17}$. Certes, en Angleterre, cette origine française des Brunel n'est pas ignorée, mais elle n'est que rapidement évoquée, surtout pour étayer la comparaison avec les Stephenson. Voici ce qu'écrivait déjà, par exemple, J. F. Layson dans son ouvrage de 1862 consacré aux pionniers du rail : « The Stephensons were skilful and ingenious, practical and shewd. The Brunels, original and daring, idealistic and ambitious. The former father and son may be justly regarded as true representatives of the English school of engineering; the latter as bold exponents of the Continental » ${ }^{18}$ ( « Les Stephenson étaient pleins de talents et ingénieux, pratiques et rusés. Les Brunel, originaux et audacieux, idéalistes et ambitieux. Les premiers, père et fils, peuvent à juste titre être considérés comme les véritables représentants de l'école anglaise d'ingénierie. Les seconds comme des propagateurs résolus de l'école continentale »). En se servant ainsi de l'origine française des Brunel dans une optique comparative, les biographes anglais n'ont cependant pas apporté beaucoup d'informations concrètes sur leur éducation, les relations qu'ils ont pu entretenir avec la France, ne retenant finalement qu'un petit nombre de faits plus ou moins exacts et inlassablement répétés. Pour A. Buchanan notamment, les choses sont claires : « Marc became British, but Isambard Kingdom was British from birth ... He was British in everything except his name, and he appears to have been regarded by most of his con-

York, 1953. Bernard Dumpleton et Muriel Miller, Brunel's three ships, Melksham, 1974 ; Denis Griffiths, Andrew Lambert, Fred Walker, Brunel's ships, Londres, Chatham Publishing, 1999.

17 Citons par exemple la biographie de Frédéric Passy, Le petit poucet du XIXème siècle. Georges Stephenson et la naissance du chemin de fer, 1910.

18 J. F. Layson, The Stephensons and Other Railway Pioneers, Londres, 1862, p. 297. 
temporaries... " ${ }^{19}$ (« Marc devint britannique, mais Isambard Kingdom était britannique de naissance ... II était britannique en tout, excepté son nom, et était considéré comme tel par la plupart de ses contemporains... »).

Les réalisations concrètes des Brunel ne doivent, il est vrai, pratiquement rien à la France. Elles se situent pour la quasi-totalité en Angleterre. Mais cela n'exclut pas I'influence qu'ont pu avoir leur éducation et leur culture partiellement françaises dans la façon d'aborder leur métier d'ingénieur, un esprit assez théorique ne dédaignant pas le recours aux mathématiques, une pratique du dessin également beaucoup plus développée que chez la plupart de leurs concurrents britanniques, une façon enfin d'envisager l'innovation, qui tout en étant pragmatique, s'avérait détachée des résultats économiques immédiats. Les liens qu'ils ont pu entretenir avec leur pays d'origine liens très directs pour le père, plus distants, il est vrai, pour le fils - constitués d'échanges d'informations, de collaborations projetées ou effectives, firent qu'ils prirent leur part dans les transferts de techniques qui existèrent alors entre l'Angleterre et la France, et contribuèrent ainsi à constituer une ingénierie véritablement européenne. Ce sont ces liens cachés et leurs conséquences, curieusement très peu étudiés, que I'on se propose de faire ressortir dans cet article, sachant que les sources disponibles sont loin d'avoir été toutes exploitées et qu'il reste sûrement beaucoup à découvrir et à dire sur un sujet qui s'avère étonnant à plus d'un titre.

\section{Un apprentissage franco-américain}

Marc Isambard naquit le 25 avril 1769 à Hacqueville, un village du Vexin normand, proche d'Etrépagny et de Gisors. Sa famille appartenait au petit milieu fermé des gestionnaires domaniaux de cette riche région céréalière dont la plupart des propriétaires résidaient à Rouen ou Paris. Le père de Marc Isambard était ainsi fermier et régisseur des Le Couteulx du Molay, seigneurs du lieu, banquiers à Rouen et à Paris ${ }^{20}$. Il avait épousé en premières noces Marie-Anne Millard, la fille du receveur de la baronnie voisine d'Etrépagny, propriété depuis 1752 du Président Turgot, cousin du futur contrôleur général des finances. Celle-ci étant décédée en 1765, Jean-Charles se

19 A. Buchanan, Brunel, op. cit, p. 7. Concernant Marc Isambard, il ajoutait : " His father, it is true, has been born, bred and educated in France, and could thus have been regarded as genuinely French » («Son père, il est vrai, était né et avait été élevé et éduqué en France, et peut ainsi être considéré comme authentiquement français »).

20 A. Ancourt, Hacqueville (Eure). Histoire d'une paroisse rurale du Vexin Normand. Des origines à 1900, 1964, p. 237-239; Jean-Jacques Pilinski, "Les Brunel », Les Cahiers de la S.H.B.E., $n^{\circ} 31,1993$, p. 3-29. remaria en 1766 avec Angélique Victoire Lefebvre qui lui donna sept enfants dont trois survécurent. L'aîné Ange Charles (1767-1833) prit la succession de son père comme fermier et receveur de la terre d'Hacqueville, et fut même longtemps maire de sa commune. Marc Isambard, quant à lui, après avoir été envoyé à huit ans au collège de Gisors, fut placé à onze ans en 1780 au séminaire Saint Nicaise de Rouen ${ }^{21}$. Son peu d'attrait pour la prêtrise décida son père à le mettre en pension toujours à Rouen chez un parent, François Carpentier, ancien capitaine au long cours devenu négociant, avec l'idée d'en faire un marin. II eut alors la chance de pouvoir suivre au collège de Rouen le cours public d'hydrographie de Vincent Dulague (1729-1805), I'un de ces cours institués par Colbert pour former les marins dans les villes portuaires ${ }^{22}$. Excellent pédagogue, Dulague vit son cours édité dès 1768 sous le titre Leçons de Navigation et utilisé par toutes les écoles d'hydrographie. Le jeune Marc Isambard put ainsi s'initier à la géométrie, à l'astronomie, à la mécanique. Pour les travaux pratiques, il bénéficia aussi des leçons d'un répétiteur, un nommé Fouré. II apprit encore à dessiner, s'exerçant au relevé sur les monuments de Rouen, et dès qu'il eut appris les rudiments de la trigonométrie, il confectionna un théodolite pour mesurer la hauteur de la flèche de la cathédrale de Rouen ${ }^{23}$. II manifesta, en effet, un goût précoce pour les instruments. II aurait ainsi, paraît-il, fabriqué très tôt « un instrument destiné à reproduire les sons de l'orgue, en n'employant qu'un seul tuyau » pour un camarade d'enfance, Jacques-Charles Allard, avec lequel il conserva sa vie durant des relations amicales ${ }^{24}$. Pendant les vacances à Hacqueville, il passait son temps chez le menuisier du village, et réalisa avec lui la monture d'un octant en bois, conforme au cours de Dulague, qu'il refit ensuite en bois d'ébène et qu'il utilisa constamment par la suite. II construisit aussi de nombreuses maquettes de vaisseaux dont sa chambre, paraîtil, fut rapidement décorée ${ }^{25}$. Cette formation lui permit, en tout cas, d'être autorisé à embarquer en 1786 - il avait donc 17 ans - comme Volontaire d'Honneur sur la frégate

21 Édouard Frère, « Notice historique sur la vie et les travaux de Marc-Isambart Brunel ", Mémoire de l'Académie de Rouen, 1849-1850, p. 67.

22 Né à Dieppe, il était l'élève de l'astronome Bouin. Il fut nommé au collège de Rouen en 1762, après l'expulsion des Jésuites.

23 R. Beamish, Memoir..., op. cit., p. 11, citant le passage d'une lettre à un ami français non désigné : « II (V. Dulague, à qui il en aurait fait la proposition après trois cours de trigonométrie) l'admit. Je fis de suite un instrument, assez grossier à la vérité, mais assez juste, pour confirmer la théorie et la pratique ».

24 É. Frère, "Notice historique... », op. cit., p. 69.

25 R. Beamish, Memoir..., op. cit., p. 12 (qui précise, par ouï dire, avoir été d'une singulière beauté de forme et de finesse d'exécution). 


\section{Les Brunel père et fils : deux célèbres ingénieurs anglais « Made in France »}

Maréchal de Castries (du nom du Ministre de la Marine). II navigua à son bord durant six ans jusqu'au licenciement de l'équipage en janvier $1792^{26}$, principalement du côté des mers Caraïbes sans qu'on sache précisément ce qu'il fit et apprit. La seule chose qu'il conserva de ces voyages, fut un dessin confectionné en 1790 à la Guadeloupe pour une machine à décortiquer le café27.

Étant à Paris début 1793 pour s'occuper d'obtenir un passeport du Ministre de la Marine destiné à l'achat de grains et de farines en Amérique ${ }^{28}$, il aurait eu des démêlés avec des Montagnards lors d'une réunion tenue le 16 janvier au Café de l'Échelle proche du Palais Royal à propos du procès de Louis $\mathrm{XVI}$, qui I'amenèrent à quitter précipitamment la capitale. II se réfugia en Normandie, et le 7 juillet, réussit à s'embarquer sur un navire américain, le Liberty. Arrivé à New-York le 7 septembre, il gagna tout de suite Albany, dernier port sur l'Hudson accessible aux bateaux de mer, où il retrouva deux compagnons de traversée Pierre Pharoux et Simon Desjardins. Il est difficile de suivre les biographes lorsqu'ils prétendent que cette rencontre était un hasard. Ces deux hommes étaient, en effet, les agents d'une Compagnie dite de New York et qui prit bientôt le nom de Castorland, créée à Paris le 28 juin 1793 avec pour objectif de prendre concrètement possession de 220000 acres de terre situées au nord-est de l'Etat de New-York, sur la rive droite de la Black River jusqu'au lac Ontario, acquises en août 1792 par Pierre Chassanis, pour les lotir en vue de créer une colonie de peuplement ${ }^{29}$. La Compagnie comptait intéresser les émigrés qui, fuyant la Révolution, souhaitaient s'établir aux Etats-Unis. Pharoux et Desjardins, ses agents sur place, accompagnés de Marc Isambard Brunel, passèrent ainsi avec quatre Indiens les mois de septembre et octobre 1793 à faire, dans cette contrée encore sauvage et en couchant sous la tente, I'arpentage méthodique du territoire en vue du lotissement ${ }^{30}$. Comment ne pas

26 Selon P. Clements (Marc Isambard Brunel, op. cit., p. 7), alors que R. Beamisch situe ce licenciement début 1793.

27 R. Beamisch, Memoir..., op. cit., p. 15.

28 É. Frère, « Notice historique... », op. cit., p. 72.

29 Sur cette histoire extraordinaire : Edith Pilcher, Castorland. French Refugees in the Western Adirondacks 1793-1814, Harrisson, Harbor Hill Books, 1985. Étude fondée sur l'analyse du Journal de Castorland, manuscrit de 706 pages, découvert à Paris en 1862 par un jeune américain, William Appleton, et conservé aujourd'hui à la Massachussetts Historical Society (Boston). Le vrai promoteur de l'entreprise était le beau frère de Chassanis, le financier d'origine nantaise Jacques-Donatien Le Ray, qui avait accueilli Benjamin Franklin à Passy en 1777.

30 Voici comment É. Frère racontait les choses en 1849 (op. cit., p. 72-73) : «Deux de ses compagnons de traversée, dont I'un était M. Pharoux, un habile architecte de Paris, accueillirent I'offre que leur fit Brunel non seulement de les accompagner, imaginer que Marc Isambard ait dès son séjour à Paris début 1793 projeté de se lancer dans cette aventure, ce qui expliquerait sa demande de passeport pour I'Amérique? I| conserva en tout cas un souvenir très présent de cette expédition, et dans les lettres qu'il écrivit entre autres à son ami J. C. Allard dans les années 1830, il expliqua avec force détails combien cette expérience avait été pour lui inoubliable et fondatrice ${ }^{31}$.

La Compagnie Castorland poursuivit ses travaux, mais I'année suivante, en 1794, toujours à l'instigation de Pierre Pharoux, Marc Isambard se trouva engagé dans une autre entreprise, commanditée par un homme d'affaires new-yorkais, John Thurman ${ }^{32}$. II s'agissait cette fois de reconnaître le tracé d'un futur canal destiné à relier l'Hudson au lac Champlain, en fait de vérifier la pertinence des études entreprises par la Northern Inland Lock Navigation, une compagnie créée en 1792 à I'initiative du général Philip Schuyler, 17331804, héros de l'indépendance devenu sénateur, beau père d'Alexandre Hamilton, le secrétaire du Trésor. Elle

mais de leur servir de capitaine dans cette lointaine et difficile expédition. Partant au nombre de sept, quatre naturels du pays, que Brunel s'étaient prudemment adjoints, et trois Français, ils allaient ainsi lever les plans et prendre possession, au nom d'une compagnie française, d'immenses terrains dont l'étendue était d'environ 220000 acres. Ils savaient que ces terrains étaient situés à plus de $400 \mathrm{~km}$ de New-York, compris entre le $44^{\circ}$ degré de latitude et le cours de la Black River. Ils emportaient avec eux deux tentes, et, confiants dans ces simples indications, contents de la richesse de leur matériel, ils entreprenaient cette aventureuse expédition en septembre et octobre 1793 au milieu d'une contrée inhabitée, presque sauvage, dans un pays dont le sol, la végétation, les accidents leur étaient complètement étrangers..." ".

31 Arch. dép. Eure, III F, 488, lettre à Allard du 16 septembre 1831 : «Lorsque je naviguais sur le lac Ontario en 1793, nous n'y rencontrions que quelques Indiens, c'est-à-dire des sauvages. Partout, nous couchions sous la tente. Venant d'échapper au gouvernement Robespierre, nous (trois français) et quatre américains trouvions une contrée délicieuse, malgré que nous eussions à rencontrer des ours qui après tout sont moins féroces que les agents du despote; sans inquiétude, sans aucune relation avec aucun Blanc, nous nous disions souvent que faiton ? ... Nous étions des Robinsons, j'étais le capitaine du bateau, car ce n'était que par ce moyen que nous avions pu être muni de provisions. Tous les soirs, c'est-à-dire vers les 5 heures, nous choisissions quelque position favorable pour monter les tentes: une pour nous et une pour nos gens. Pendant ce temps, ils coupaient et débitaient un arbre avec une adresse dont on n'a pas d'idée parmi les Européens. Nous avions bientôt un grand feu, un bâton servait de crémaillère. La marmite, la broche étaient bientôt en activité... ».

32 Roger G. Kennedy, Orders from France. The Americans and the French in a Revolutionary World 1780-1820, New York, Alfred A. Knopf, 1989, p. 54. 
associait des capitalistes new-yorkais possessionnés le long du cours de l'Hudson qui y voyaient, entre autres, I'occasion de réaliser de belles plus values foncières ${ }^{33}$. J. Thurman, qui ne faisait pas partie de cette compagnie, était alors engagé plus au nord dans le développement d'une vaste zone comprise entre les monts Adirondacks et les lacs George et Champlain. II avait fondé en 1792 sur la rive ouest de la haute vallée de l'Hudson une ville portant son nom, à laquelle il ajouta en 1799 celles de Bolton et Chester. Pharoux et Brunel se virent aussi entraînés dans la remontée de la rivière Mohawk - la rivière des Iroquois - et à reconnaître à la suite le cours peu profond du Wood Creek, cours d'eau qui plus tard formeront partie du réseau du canal Érié et qui, alors, faisait l'objet d'études et travaux de la part de la Western Inland Lock Company, autre entreprise patronnée par Schuyler ${ }^{34}$. Marc Isambard s'acquitta visiblement bien de cette tâche: «II fallut que Brunel mit en œuvre cette fécondité inépuisable de ressources, qui devait être un des caractères de son génie ; il fallut en un mot qu'il devint ingénieur » relata Édouard Frère en 1849, à propos précisément de cette expédition sur la Mohawk, en s'appuyant visiblement sur ce que Marc Isambard lui avait personnellement raconté ${ }^{35}$. Le fait est que cette expérience de terrain lui donna forcément I'occasion de mettre en pratique l'enseignement de Dulague topographie, mécanique... - et sans doute aussi ce qu'il avait commencé à faire lorsqu'il était jeune marin ${ }^{36}$.

33 Sur les premières entreprises de canaux de la région de NewYork : Laurence M. Hauptman, "Command Performance: Philip Schuyler and the New York State-Oneida Treaty of 1795 », dans L. Gordon McLester III, L. M. Hauptman éd., The Oneida Indian Journey, From New York to Wisconsin 1784-1860, Madison, The University of Wisconsin Press, 1999, p. 38. Voir aussi The Inland Navigation Surveys of 1792, New York State Museum, 1992.

34 Nathan Miller «Private entreprise in inland navigation: the Mohawk Route Prior to the Erie Canal », New York History, 31, octobre 1950, p. 398

35 Frère, op. cit., p. 75 . Dans une lettre du 27 juin 1834 publiée par Jean-Jacques Pilinski («Les Brunel... », op. cit., p. 6), M. I. Brunel écrivait encore: « Mon voyage dans I'intérieur, à l'arrivée à New-York, me fit ingénieur parce qu'il fallait constamment surmonter quelques difficultés et pourvoir pour l'avenir et même seulement pour le retour. Un de mes compagnons de voyage, un très habile architecte de Paris, me croyait ou me prenait toujours pour un ingénieur, ou enfin me considérait comme tel : aussi me proposa-t-il pour coopérer avec lui relativement à une ligne de canal d'Albany jusqu'au lac Champlain... ».

36 P. Cléments, en se fondant sur un dessin retrouvé dans une collection privée, la collection Hurst (p. 15), a prétendu qu'il aurait dès cette époque conçu des machines pour extraire les charges de la rivière. É. Frère est plus prudent «Ayant à débarrasser ces rivières obstruées de masses de rochers, d'arbres
On sait malheureusement peu de choses sur Pierre Pharoux ${ }^{37}$. Architecte parisien, il avait été pourvu d'un office d'expert juré en janvier 1789, ce qui atteste déjà une certaine expérience ${ }^{38}$, et s'était intéressé à l'architecture hospitalière ${ }^{39}$. II était vraisemblablement lié aux Leray de Chaumont, les vrais promoteurs de Castorland, qui avaient été même sans doute responsables de son engagement ${ }^{40}$. En même temps qu'il travaillait à l'affaire Castorland, Pharoux développa, du reste, autour de New-York une activité remarquable d'architecte ${ }^{41}$. Elle s'exerça d'abord au service de la famille Livingston, puissante famille américaine, chef du parti français à New-York, très liée d'ailleurs aux Le Ray. La principale figure était alors le chancelier Robert Livingston (17461813), futur ambassadeur des Etats-Unis à Paris (de 1801 à 1804). Les Livingston possédaient de grands domaines sur l'Hudson, autour de Clermont au sud d'Albany. Pharoux réalisa pour eux un certain nombre de projets, tant pour des maisons à New-York, à Broadway, que pour des maisons de campagne autour de Clermont, en particulier celle dite Masséna, construite pour John, le frère de Robert ${ }^{42}$. Pharoux fut aussi urbaniste et conçut le plan de petites villes nouvelles à établir le long de

séculaires déracinés par les ouragans, et n'ayant comme seul moyen d'exécution que la hache, le seul outil dont se servent les habitants dans cette contrée boisée... », op. cit., p. 75.

37 II semble qu'il soit né à Passy en novembre 1759.

38 Journal de Paris, 14 janvier 1789. II était membre de l'assemblée électorale de Paris du 18 novembre 1790. Assemblées électorales de Paris, 18 novembre 1890 - 12 août 1792 : publiées d'après les originaux des Archives nationales, Paris 1890. Étienne Pierre Pharoux, juré expert, 32 ans, 15 rue de Cléry. ॥ signa en 1790 comme juré expert des plans rue de Boulainvilliers et de Passy, Archives nationales (Paris) [ensuite AN] NIIISeine, 936. 39 M. Pharoux, architecte, Mémoire sur les hôpitaux à construire, Paris, 1787.

40 Jacques Donatien (1725-1803) et son fils Jacques Donatien, dit James (1760-1839). Le premier fut le principal financier des insurgés américains durant la guerre d'Indépendance, il leur fournit armes, poudres et munitions, logea Benjamin Franklin de 1777 à 1785 dans son hôtel de Passy. Le second, émigré aux Etats-Unis avant la Révolution, prit la nationalité américaine, lorsqu'il épousa Grace Cox héritière d'une riche famille du New Jersey. C'est son beau frère Pierre Chassanis, époux de Marie Sophie Leray qui acquit en août 1792 les 220000 acres de l'opération Castorland. Thiéry Claeys, Dictionnaire biographique des financiers en France au XVIIlème siècle, Paris, Editions SPM, 2008.

41 Ce qui suit s'inspire très largement de R. G. Kennedy, Orders from France, op. cit.

42 Ibid., p. 63 L'auteur fait même I'hypothèse que P. Pharoux aurait préparé des plans pour la célèbre demeure de Robert Livingston, appelée Arryl House à Clermont, avant son départ de France, et que c'est une des raisons pour laquelle il serait venu en Amérique. 


\section{Les Brunel père et fils : deux célèbres ingénieurs anglais « Made in France "}

I'Hudson dans l'esprit des architectes français à la fois néo-classiques et utopistes, tels Étienne-Louis Boullée et Claude-Nicolas Ledoux dont il avait peut-être été l'élève. Il dressa ainsi non seulement les plans pour Cartorland d'une ville nouvelle, appelée Castorville, mais aussi ceux d'un village appelé Tivoli, situé au sud de Clermont, pour un Français Pierre de La Bigarre (dont le vrai nom serait Pierre de Sauvigard), réfugié de Saint-Domingue, devenu cousin par alliance de Robert Livingston ${ }^{43}$. Le plus intéressant reste cependant le projet conçu début 1795 pour les Livingston à côté de l'ancienne ville d'Athens (au sud de Albany). Des dessins de cette ville nouvelle nommée Esperanza ont été conservés, ils nous montrent un plan général d'une géométrie parfaite et l'élévation de différents bâtiments, un marché, une auberge, un cityhall d'allure néo-classique et une église à portiques ${ }^{44}$.

Pharoux forma, en tout cas, Marc Isambard à l'architecture, l'employant sans doute comme dessinateur pour certains de ses projets, ce qui l'aurait même conduit à réaliser seul un projet pour le Palais des Congrès à Washington, ce qui est peu vraisemblable ${ }^{45}$. II est plausible, en revanche, que Marc Isambard ait fait une proposition début 1795 pour la construction d'une salle de salle de Théâtre à New-York ${ }^{46}$. Le destin cependant

43 Ibid., p. 77. Le Chancelier Robert Livingston et Pierre de La Bigarre étaient tous deux membres de la Society for the Promotion of Agriculture, Arts and Manufactures, basée à Albany. Ils travaillèrent ensemble en 1799 à un nouveau procédé de fabrication du papier. R. Livingston, "A new discovery relative to the Art of Manufacturing Paper, 8 septembre 1799 », Transactions of the Society for the Promotion Agriculture, Arts and Manufactures, vol. 1, 1801.

44 Ibid., p. 70. L'existence de ces dessins est attestée par le Journal de Castorland. Ils ont été gravés par Charles B. J. Fevret de Saint-Memin. On les trouve à la Corcorian Gallery de Washington et à la State Library of New York. P. Pharoux travailla aussi début 1795 à un projet de ville nouvelle à Fort Stanwix sur la rivière de la Mohawk pour le marchand new-yorkais Dominick Lynch, le fondateur de la ville de Rome. L'idée était aussi d'y établir un entrepôt pour la compagnie de Castorland.

45 La thèse a été défendue par R. Beamish (Memoir..., op. cit., p. 32), et reprise par P. Clements qui fournit à l'appui un dessin trouvé dans la collection Hurst (p. 15). Elle est contredite par R. G. Kennedy, qui fait remarquer que le concours était antérieur à l'arrivée de P. Pharoux et M. I. Brunel en Amérique. R. Beamish soutient, en outre, que $P$. Pharoux aurait présenté un projet séparé. 46 Voir sa version des faits telle qu'elle apparaît dans la lettre du 27 juin 1834 citée note 35 : «Pendant son absence de NewYork (il s'agit de Pierre Pharoux), on demanda des plans pour la première salle de la ville, les miens furent adoptés et exécutés. Mon ami apprit ce choix lorsqu'il était sur les bords de la rivière nommée Black River près du lac Ontario. J'ai de lui une lettre datée des grandes chutes de Black River, où il me dit qu'il félicite les propriétaires du choix qu'ils ont fait...; ensuite dans sa bouleversa les choses: Pharoux retourné œuvrer à Castorland en juin 1795, se noya avec sept compagnons le 21 septembre suivant, en cherchant à traverser la Black River à Long Falls. Ayant perdu ainsi son mentor, Marc Isambard continua à s'occuper d'architecture. II aurait réalisé alors un projet pour une banque à Wall Street ${ }^{47}$, et travailla pour un autre personnage connu de I'histoire américaine, Aaron Burr (1756-1839), alors procureur général de l'Etat de New-York, pour lequel il dessina un projet de façade baroque destinée à sa résidence de Marble Hill à Manhattan ${ }^{48}$. Il est vraisemblable surtout qu'à ce moment, il devint le collaborateur d'un autre architecte français Joseph Mangin qui, arrivé aux Etats-Unis en 1793, s'associa courant 1794 avec I'architecte new-yorkais John McComb Jr. pour construire I'Equestrian Circus sur Greenwich Street ${ }^{49}$. Tous deux réalisèrent ensuite le Park Theather (à Park Row au sud de Manhattan), peut-être à partir du projet de Brunel simplifié par mesure d'économie et en l'associant à la réalisation ${ }^{50}$. Puis, ils construisirent en 1796 une prison fédérale à Greenwich Village, considérée comme prison modèle, et plus tard le City Hall de New York (à partir de 1802) et l'ancienne cathédrale Saint-Patrick (18091815). Joseph Mangin fut, par ailleurs, nommé en 1796 I'un des trois « surveyors» de New-York, ce qui l'amena à travailler au plan quadrillé de l'extension de la ville au nord de l'ancienne ville hollandaise et à s'occuper, entre autres, de la construction d'entrepôts et de fortifications ${ }^{51}$. II est tentant d'établir un rapprochement entre cette prise de fonction et l'obtention par Brunel

lettre, il me dit : réservez-moi quelques chiffons à peindre sur vos décorations, afin que j'ai mon entrée franche... ». On apprend aussi que les Livingston figuraient parmi les propriétaires de cette salle. Cette lettre que M. I. Brunel avait conservée était datée du 20 septembre 1795, veille de la mort de P. Pharoux.

47 Dessin de la collection Hurst publié par P. Clements, Marc Isambard Brunel, op. cit. p. 15.

48 R. G. Kennedy, Orders from France, op. cit, p. 79. L'auteur en profite pour souligner que $A$. Burr fréquentait Marc Isambard en 1811-1812 lors de son exil à Londres, après son bannissement suite au duel mortel qui l'avait opposé en 1804 à Alexandre Hamilton. Ils discutaient, entre autres, de mécanique.

49 R. G. Kennedy, Orders from France, p. 112-113. La biographie de Joseph Mangin reste imprécise. II devait être fils ou neveu de I'architecte parisien Charles Mangin (1721-1807), auteur en 1790-92 d'un projet présenté à l'Assemblée nationale de transformation du Louvre en Chambre des députés et de diverses propositions pour l'embellissement de Paris. Voir Pétition du sr Mangin père, architecte, et supplément au Mémoire instructif dont il a fait hommage à l'Assemblée constituante en avril 1791, 20 janvier 1792.

50 Ibid., p. 112. Alois M. Nagler, A source Book in Theatrical History, Dover publications, New-York, 1952.

51 R. G. Kennedy, Orders from France, op. cit., p. 112. 
de la citoyenneté américaine en juin 1796, suivie de sa nomination dès septembre comme "chief ingenior » (ingénieur en chef) de la ville de New-York, bien que celle-ci, signalée par tous les biographes britanniques, ne soit confirmée par aucun document original. Frère avait, d'ailleurs, été beaucoup moins précis, se contentant d'écrire: «Faut-il exécuter des fortifications pour la défense du premier port des Etats-Unis, défense confiée à un colonel français du génie ? Brunel est réclamé et employé avec distinction à ces travaux, au nombre desquels il faut comprendre l'établissement d'un arsenal et d'une fonderie de canons ${ }^{52}$. $\|$ est clair, en tout cas, qu'à partir de ce moment, Marc Isambard s'est livré à un véritable travail d'ingénieur, se consacrant notamment à l'élaboration de machines, destinées entres autres à l'arsenal. "L'ingénieux mécanisme qu'il imagina pour exécuter l'opération de forage de canons, ses nouveaux alésoirs ..., une foule d'inventions et d'idées fécondes qu'il mit au jour, suffiraient pour établir sa célébrité » ajoutait Frère en 1849. Le colonel du génie auquel il fait allusion, pourrait être Anne-Louis de Tousard (17491817), ancien officier d'artillerie français qui avait servi à Saint-Domingue, et prisonnier en 1794 des forces haïtiennes avait pu passer aux Etats-Unis et rejoindre le Corps of Artillerists and Engineers, nouvellement créé pour s'occuper entre autres jusqu'en 1802 de la construction de nouvelles fortifications (West Point, Newport à Rhodes Island...) et de l'amélioration de la fonte et du forage des canons, en s'appuyant sans doute sur la Description de l'Art de fabriquer les canons de Gaspard Monge publié en 179453. S'il ne serait pas surprenant que Marc Isambard ait été associé à ces travaux, son champ d'investigation devait être plus large, puisque ce serait dès cette époque qu'il aurait conçu le principe de la machine à fabriquer

52 É. Frère, "Notice historique... », op. cit., p. 76. On peut s'interroger sur l'endroit où se trouvait cet arsenal et cette fonderie. S'agissait-il de Governors Island au sud de Manhattan (Fort Jay) ou encore d'une fonderie déjà établie à Brooklyn. R. Beamisch qui évoque aussi cette fonderie de canons, en ajoutant qu'il n'en existait pas auparavant aux Etats-Unis (op. cit., p. 34), signale plus loin (p. 38) la présence de Marc Isambard à Fort Montgomery, sur l'Hudson, près de West Point. La dite fonderie a pu aussi être établie à proximité, sachant cependant que la fonderie de West Point n'aurait été créée qu'en 1817.

53 Meritt Roe Smith, Military entreprise and technological change: perspectives on the American Experiences. 1985, p. 45. A. L. de Tousard publia un ouvrage qui servit longtemps de référence aux Etats-Unis: American artillerist's companion or Elements of artillery, Treating of all the kinds of firearms, in detail, and of the formation, object and service of the flying or horse artillery, preceded by an introductory dissertation on cannon, Philadelphie, 1809. les poulies qui allait bientôt le rendre célèbre ${ }^{54}$. En tout cas, c'est visiblement la possibilité de mettre en pratique des savoirs appris à Rouen et au service de la Marine française qui firent de lui, comme il l'expliqua lui-même par la suite, un expert polyvalent - mécanicien, ingénieur, architecte -, au contact d'autres français, tels Pharoux, Mangin et de Tousard, venus comme lui chercher dans le nouveau monde des opportunités d'entreprendre.

\section{Retour en Europe}

Les raisons pour lesquelles Marc décida de rentrer en Europe, en s'embarquant sur l'Haliphax le 20 janvier 1799, ont donné lieu à diverses hypothèses. Les biographes britanniques ont souvent mis en avant son désir de rejoindre Sophie Kingdom, une jeune Anglaise qu'il avait connue à Rouen et qu'il épousa, en effet, dès novembre $1799^{55}$. On a aussi écrit que Marc Isambard espérait y rencontrer un plus grand intérêt pour ses inventions mécaniques. La tradition rapporte ainsi qu'il aurait été informé lors d'un dîner chez Alexandre Hamilton par le Français de La Bigarre, I'homme de Tivoli, tout juste rentré d'un voyage en Angleterre, des besoins de modernisation de la Marine anglaise, notamment pour la fabrication des poulies et qu'A. Hamilton l'aurait encouragé à tenter sa chance en lui donnant une lettre de recommandation pour le Ministre de la Marine, Lord Spencer. Deux autres hypothèses sont cependant sorties d'archives américaines : Marc Isambard aurait été envoyé en Angleterre par Robert Livingston pour s'occuper d'un projet de navigation à vapeur (car avant de devenir en 1809 l'associé de Robert Fulton pour la navigation sur I'Hudson, il s'était intéressé à la question dès 1798)56, à moins, pour finir, qu'il n'ait fui l'Amérique pour cause de difficultés financières ${ }^{57}$.

54 R Beamisch, Memoir..., op. cit., p. 38.

55 É. Frère, «Notice historique... », op. cit., p. 76. P. Clements, Marc Isambard Brunel, op. cit, p. 18.

56 R. G. Kennedy, Orders from France, op. cit, p. 82. Dès 1798 , il s'était entendu avec John Stevens (1749-1838) et J. Roosevelt fondeur dans le New-Jersey pour la construction d'un bateau à vapeur. Cynthia Owen Philip, « Robert R. Livingston. Enthusiastic Inventor, Prudent Entrepreneur », dans The Livingston Legacy, Three century of American History, Symposium, 6-7 juin 1986. 57 R. G. Kennedy, Orders from France, op. cit., p. 82. Le soupçon vient d'une lettre écrite en 1812 par le neveu de John Thurman évoquant une dette non honorée pour un prêt en 1799 qui aurait permis à Marc Isambard d'éviter la prison. L'auteur pense d'ailleurs que Marc Isambard continua à travailler après1794 pour J. Thurman, entre autres pour la construction de moulins, scieries, distilleries ainsi que d'un moulin à foulon transformé en filature de coton en 1797 à laquelle fut adjointe une fabrique d'impression de calicots.

Évoquons enfin une dernière hypothèse qui concerne peut- 


\section{Les Brunel père et fils : deux célèbres ingénieurs anglais « Made in France »}

Installé en Angleterre, Marc Isambard ne fit pas, comme on aurait pu le penser, immédiatement affaire avec la Marine. II commença par s'employer à faire valoir ses talents de mécanicien en proposant plusieurs types de machines. La première fut une sorte de pantographe, dite autographe, destinée à copier les dessins, les cartes et même les écritures commerciales qu'il breveta dès avril $1799^{58}$. II mit encore au point une machine à tordre les fils de coton pour les mettre en balles, une autre à battre les cartes, une, enfin, brevetée en novembre 1802 pour fabriquer les lisières de toiles fines de coton telles que mousselines et batistes ${ }^{59}$. Sa grande œuvre fut, cependant, la conception et l'installation d'un atelier de fabrication en série de poulies destinées à l'équipement des navires - un vaisseau de 74 canons en réclamait $922^{60}$. Pour parvenir à installer dès 1803 ses premières machines à Portsmouth, Marc Isambard bénéficia de deux appuis essentiels, celui du Samuel Bentham, I'Inspecteur général des travaux de la Navy, qui avait lui-même réfléchi à la conception de telles machines et qui eut le grand mérite de lui accorder toute sa confiance ${ }^{61}$, et celui

être une période ultérieure, avancée par Louis Constant Wairy, premier valet de Napoléon : «Cet homme si justement apprécié, admiré en Angleterre, avait voulu consacré ses talents à sa patrie. Il fut repoussé par Bonaparte, et obligé de porter son industrie et son génie parmi les Anglais... », Mémoires de Constant, 1830, p. 109. Elle est évoquée dans un article normand paru en 1849, au moment de son décès: "Le célèbre ingénieur Brunel dont nous avons hier annoncé la mort ... était né dans le département de l'Eure à Hacqueville en 1769. II était fils d'un fermier ; il avait une sœur qui épousa Mr Langlois, longtemps maire d'Ecouis... Mr Brunel avait, il paraît, offert tout d'abord son invention à la France (la machine à fabriquer les poulies), mais on ne lui accorda pas le prix auquel il l'estimait, et il la porta chez les anglais... ». Arch. dép. Eure, III F, 488.

58 É. Frère, "Notice historique... », op. cit., p. 76. Harold Bagust, The greatest Genius: a biography of Marc Isambard Brunel, Hersham, lan Allan Publishing, 2006, p. 22. L'auteur publie en annexe la liste et le contenu d'une quinzaine de brevets pris par M. I. Brunel entre 1799 et 1825.

59 R. Beamisch, Memoir.., op. cit., p. 44.

60 L'affaire a fait l'objet de nombreuses publications. Voir surtout, J. Coad, The Portsmouth Block Mills..., op. cit.

61 Charles Dupin (1784-1873) qui connaissait S. Bentham rappela ce fait lors de l'Exposition Universelle de Londres de 1851 : « L'arsenal de Portsmouth doit de grands travaux hydrauliques au général Samuel Bentham. II avait imaginé le moyen d'exécuter les poulies par les moyens mécaniques. Un Français, pauvre émigré, qui n'avait encore aucune réputation, lui communique ses inventions sur le même objet. Bentham les étudie et, sans hésiter, en reconnaît la supériorité. Loin de faire servir sa puissance pour écarter son rival, il met un zèle généreux à faire préférer l'étranger, l'inconnu ; et cet inconnu, c'était Brunel », Travaux de la Commission française. Introduction par M. le baron Charles Dupin, Exposition Universelle de 1851, p. 137. du grand mécanicien Henry Maudslay qu'il avait connu dès l'été 1799, grâce à un autre émigré français, de Bacquencourt. Ensemble, ils confectionnèrent d'abord des modèles qui permirent d'obtenir l'accord de l'Amirauté, puis les machines grandeur nature, en s'aidant $d u$ chariot à tour que $\mathrm{H}$. Maudslay avait mis au point peu de temps auparavant. Cette réalisation, qui permit à la Navy de rénover en un temps record sa flotte juste avant Trafalgar, assura la gloire de Brunel comme ingénieur. On loua de toutes parts la parfaite organisation de la ligne de fabrication qui mettait en œuvre pas moins de 44 machines différentes, véritables machines-outils ${ }^{62}$. Elle suscita aussi des innovations : scie circulaire, machines à courber les bois, à scier les bois de placage... Fort des succès enregistrés à Portsmouth, Marc Isambard reçut mission d'installer une scierie dans l'arsenal de Woolwich en 1808, puis dans celui de Chatham en 1812. Dès 1807, il avait aussi créé sa propre entreprise, équipée de grandes scies circulaires, à Battersea au sud-ouest de Londres en face de Chelsea où il avait établi sa résidence. Elle travaillait pour les marines de commerce, notamment les Compagnies des Indes. En 1810, il y ajouta un atelier de fabrication mécanique de bottes et souliers à usage militaire, cloués en série selon un système spécialement breveté en octobre de la même année ${ }^{63}$.

Tant que l'Angleterre était en guerre contre la France, Marc Isambard n'avait entretenu sans doute que des relations discrètes avec son pays d'origine. Mais dès octobre 1814, cinq mois donc après l'abdication de Napoléon sur l'île d'Elbe, il prit depuis Calais le chemin de Paris, et profita du voyage pour aller rendre visite à Liancourt au duc François Alexandre Frédéric de La Rochefoucauld (1747-1827) qu'il avait peut-être connu aux Etats-Unis ${ }^{64}$. Apparemment, il

62 Ibid., "Le mouvement général de 44 mécanismes coordonnés est imprimé par une machine de 32 chevaux... Tout s'exécute avec une précision mathématique; et cela par de véritables machines-outils, que les Anglais imiteront plus tard dans les industries civiles, pour scier, tourner, perforer, planer, etc. ». 63 Cette installation est, à notre connaissance, la première réalisation de M. I. Brunel à avoir été commentée en France : « II existe près de Londres un établissement fort intéressant, dirigé par un Français nommé Brunel, établi depuis longtemps en Angleterre, et connu comme un homme de beaucoup de talents et de moyens. C'est une manufacture de souliers fabriqués mécaniquement, et dans laquelle on n'emploie que des soldats invalides, dont trente fabriquent environ 100 paires de souliers par jour ». " Note sur une fabrication mécanique des souliers », Bulletin de la Société d'Encouragement de l'Industrie nationale, XIV, 1815, p. 128.

64 Brunel Collection, The University of Bristol Library, lettre à A. Breguet, Quai de I'Horloge à Paris, adressée de Montreuilsur-Mer le 4 octobre 1814: "Je suis en chemin pour la capitale ... J'ai engagé une voiture avec trois de mes amis... ». 
se rendit ensuite au Conservatoire des arts et métiers dont La Rochefoucauld venait d'être nommé inspecteur et y rencontra Claude-Pierre Molard, son administrateur. Ils convinrent ensemble de la livraison au Conservatoire d'une machine à vapeur qu'Henry Maudslay devait fabriquer, et qui ne fut en fait jamais livrée ${ }^{65}$. Quoiqu'il en soit, ce séjour établit un contact. Des échanges d'information s'ensuivirent avec La Rochefoucauld et Molard, portant notamment sur les procédés de chauffage et d'éclairage alors en usage à Londres ou encore sur les techniques d'obtention de la fonte malléable ${ }^{66}$. L'idée de Marc Isambard était visiblement de nouer des relations professionnelles suivies avec la France, peut-être même d'y revenir, de jouer en tout cas un rôle d'intermédiaire à un moment où, face au constat de l'avance anglaise, était ressenti en France, pour l'équipement et I'industrie, un grand besoin de techniques empruntées Outre Manche. C'est apparemment dans le domaine des machines à vapeur que Marc Isambard choisit d'abord d'intervenir. ॥l avait, en fait, commencé à s'intéresser au domaine juste avant son départ pour la France en procédant à des essais sur la Tamise, sans doute dans la perspective de fournir avec H. Maudslay la Navy, et peut-être aussi parce qu'il envisageait de rééquiper l'usine de Battersea qui avait subi un terrible incendie en août $1814^{67}$. Ces investigations, on le sait, ne firent pas immédiatement suivies d'applications concrètes. Un modèle original à cylindres inclinés formant un $V$ renversé qui, dès 1816, avait été installé sur le navire postal Le Régent, assurant la liaison entre Londres et Margate sur la côte du Kent, fut finalement breveté en août $1822^{68}$. Une description illustrée en fut plus tard publiée en France dans L'Industriel, en $1827^{69}$.

Sur le duc de La Rochefoucauld, voir Charles R. Day, "Le duc de La Rochefoucauld-Liancourt, un philanthrope français (17471827) », Les Cahiers d'Histoire du CNAM, n 1, Paris, 1992, p. 45.

65 Brunel Collection, Bristol. Lettre de Chelsea du 20 janvier 1815 au duc de La Rochefoucauld : « Votre pompe à feu est en main ; mais il faut de la patience avec Maudslay qui ne néglige jamais son ouvrage... ». P. Clements, Marc Isambard Brunel, op. cit. , p. 62.

$66 \mathrm{lbid}$. , «Le gaz light ne peut convenir qu'à éclairer et pour cela il réussit parfaitement soit dans l'intérieur des manufactures ou pour les rues ... Pour en revenir aux moyens de chauffer de grands établissements, on a voulu employer la vapeur d'eau, mais on y a éprouvé de très grands inconvénients. Par le procédé en question, on ne se sert que d'air échauffé par un seul poêle hors des bâtiments; on conduit cet air sous les planchers ... ». Marc Isambard profite aussi de l'occasion pour prier le duc « d'obtenir de Mr Mollard un petit traité qu'il (lui) avait promis sur les moyens d'adoucir la fonte de fer et de la rendre malléable ... » 67 R. Beamish, Memoir.., op. cit., p. 140 ; P. Clements, Marc Isambard Brunel, op. cit., p. 62.

68 H. Bagust, The greatest Genius..., op. cit., p. 145.

69 Jacques Payen, La machine à vapeur fixe en France, Paris,
Dans les années qui suivirent son premier séjour, Marc Isambard prit deux autres initiatives en direction de la France qui, du reste, n'eurent pas plus de succès. La première, l'occasion d'un autre voyage sur le continent fin 1817, concernait la distribution d'eau potable à Paris. Marc Isambard représentait une société anglaise qui, sur le modèle de ce qui se faisait à Londres, proposait d'établir un réseau de canalisation en fonte pour distribuer l'eau dans les habitations ${ }^{70}$. On connaît, en fait, peu de choses sur ce projet si ce n'est que l'administration parisienne en refusa les conditions, confrontée, entre autres, à la résistance des porteurs d'eau ${ }^{71}$. II est possible que lors de ce second séjour, Marc Isambard ait pris aussi contact avec un nommé Louis Victor Roguin qui installa alors une scierie mécanique quartier de la Gare (actuel quartier d'Austerlitz), équipée de machines comparables à celles de l'établissement de Battersea $^{72}$ et fabriquées par le grand mécanicien parisien Etienne Calla dont on sait qu'il fit son premier séjour en Angleterre à l'automne $1817^{73}$.

Ces échanges plus ou moins fructueux avec la France furent aussi l'occasion de transferts en sens inverse. Le premier de ce genre concerna un métier à tricoter circulaire servant notamment à fabriquer les jupons que le riche industriel Guillaume Ternaux avait mis au point et installé en 1815 dans sa grande usine de Saint-Ouen ${ }^{74}$. Marc Isambard en importa le brevet en Angleterre dès mars 1816. II n'en résulta apparemment aucun débouché concret bien que Marc Isambard ait été encore en contact en 1822 sur ce sujet avec Ternaux qui lui fit parvenir un

CTHS, 1985, p. 76. « Description d'une machine à vapeur à deux cylindres inclinés employée à l'excavation du tunnel construit sous la Tamise par M. Brunel », L'Industriel, III, 1827-1828.

70 P. Clements, Marc Isambard Brunel, op. cit., p. 69.

71 Jean-Piere Dubreuil, Jean Papoul, "Les compagnies des canaux. Chronologie », Les Canaux de Paris, Paris, Délégation à I'Action Artistique, 1994, p. 108.

72 « L'établissement de M. Roguin, imité de ceux que M. Brunel, notre concitoyen, a formés dans les principaux arsenaux de la marine anglaise est le premier et même encore le seul de ce genre qui existe en France »: "Rapport fait par M. Molard jeune, au nom du Comité des arts mécaniques, sur les machines à débiter et travailler les bois, de M. Roguin ", Bulletin de la Société d'Encouragement pour I'Industrie nationale, XXI, 1822, p. 8. L'établissement que Louis Victor Joseph Marc Roguin avait créé sur la base d'un brevet pris le 5 mars 1817, fut apporté à une société en commandite le 15 décembre 1825. AN, minutier central des notaires, Et. XIV, 687.

73 «Rapport fait par Molard jeune sur la scierie mécanique de M. M. Calla », Bulletin de la Société d'Encouragement pour I'Industrie nationale, XXIII, 1824, p. 250.

74 L. M. Lohmüller, Guillaume Ternaux (1763-1833), créateur de la première intégration industrielle française, Paris, Les Editions La Cabro d'Or, 1978, p. 85,445. 


\section{Les Brunel père et fils : deux célèbres ingénieurs anglais « Made in France "}

nouveau modèle de petty-coat (jupon) $)^{75}$. Plus constructif fut ce qui se passa autour d'un procédé de traitement de surface des métaux servant à l'obtention de moiré métallique (en chauffant les tôles et en les traitant à I'acide sulfurique pour obtenir une cristallisation de surface). Le procédé avait été mis au point en 1816 par un certain Allard, installé à Paris rue Saint-Lazare qui s'en servait principalement pour traiter des feuilles de ferblanc et de cuivre ${ }^{76}$. Le procédé fut breveté en Angleterre en août 1817 par Vallet qui fit affaire avec Brunel qui lui, I'appliqua à l'étain et monta début 1818 un atelier de fabrication à Battersea ${ }^{77}$. Ces feuilles d'étain moiré connurent un certain succès pour la confection, entre autres, d'encriers, de lampes et de poêles. Un nouveau brevet fut pris en décembre 1817 et importé en France par Vallet ${ }^{78}$.

Au début des années 1820, Marc Isambard éprouva de sérieuses difficultés financières, dues, entre autres, à l'arrêt des commandes militaires. II fut même quelque temps emprisonné pour dettes, de mai à août 1821, suite à la suspension de paiement de son banquier Sykes et $\mathrm{Cie}^{79}$. II ne cessa toutefois de faire des projets, en cherchant toujours à renforcer les liens avec son pays d'origine. C'est aux techniques d'imprimerie qu'il s'intéressa alors, travaillant à l'amélioration du procédé de stéréotypie, qui utilisait au lieu de caractères mobiles des plaques sur lequel le texte était moulé en bloc ce qui convenait notamment pour l'impression sans grande qualité de journaux à grand tirage ${ }^{80}$. Marc Isambard, après avoir conclu le 11 décembre 1819, un accord d'exploitation, en cas de succès, avec le propriétaire du Times, John Walter II, prit un brevet en janvier 1820 pour Certain Improvements of Making Stereotype Plates, préconisant notamment l'emploi comme support d'une tôle d'acier ${ }^{81}$. Le procé-

75 Brunel Collection, Bristol, Journal de M. I. Brunel, 30 septembre 1822. 76 « Rapport fait par M. Cadet de Gassicourt sur le moiré métallique de M. Allard », Bulletin de la Société d'Encouragment pour I'Industrie nationale, XV, 1816, p. 210. Peut-être cet Allard était-il parent de son ami d'enfance, le notaire rouennais Jacques Charles Allard, né en 1767.

77 R. Beamisch, Memoir..., op. cit., p. 144 ; P. Clements, Marc Isambard Brunel, op. cit., p. 73. Dans cette affaire, M. I. Brunel s'associa avec un certain Samuel Shaw.

78 « Rapport fait par Mérimée sur le moiré métallique appliqué aux feuilles d'étain », Bulletin de la Société d'Encouragement pour I'Industrie nationale, XVIII, 1819, p. 15.

79 R. Beamish, Memoir..., op. cit., p. 170.

80 Ibid., p. 149 ; P. Clements, Marc Isambard Brunel, op. cit., p. 74. Sur les débuts de la stéréotypie, cf. Alain Nave, «La stéréotypie, entre innovation technique et produit éditorial », dans Alain Mercier éd., Les trois révolutions du livre, Paris, Musée des Arts et Métiers-Imprimerie Nationale Editions, 2002, p. 301. 81 R. Beamish, Memoir.., op. cit., p. 151 ; H. Bagust, The dé n'aboutit pas malgré les propositions françaises de I'éditeur du journal Le Constitutionnel, Guyet ${ }^{82}$. Sans se décourager, Marc Isambard poursuivit dans la même voie et mit au point dans la foulée une presse à copier portative (pocket copying press), servant notamment à la reproduction des lettres, brevetée en décembre $1820^{83}$. Ces travaux l'amenèrent à collaborer avec un constructeur de machines nouvellement installé à Londres, Taylor et Martineau ${ }^{84}$. II continua de travailler avec eux cette fois pour la fourniture en 1824-1825 de machines à vapeur destinées à la scierie de Battersea et au pompage des eaux du chantier du tunnel sous la Tamise (avec pompe et chaudière) ${ }^{85}$. Taylor et Martineau avaient d'ailleurs conçu avant 1824 un modèle de machine horizontale à haute

greatest Genius..., op. cit., p. 142. Comme dans le procédé traditionnel à caractères mobiles, le monobloc imprimant était moulé dans un alliage de plomb, d'étain et de bismuth. En fait, l'enjeu était plus large et concernait la mise au point de la presse rotative à laquelle M. I. Brunel manifestement travaillait. Son intérêt pour le sujet datait sans doute de discussions qu'il aurait eues avec John Walter, l'éditeur du Times, en 1814 lors des premiers essais d'impression du journal à la presse rotative effectués par Friedrich Koenig (1775-1833). Sollicité par J. Walter, M. I. Brunel aurait alors réalisé plusieurs plans pour finalement renoncer. Samuel Smiles, Men of Invention and Industry, Londres, 1884 (chap. VI).

82 Brunel Collection, Bristol. Cf. deux lettres de Marc Isambard à Breguet fils, 28 novembre 1820 : "J'ai été plus occupé que jamais d'une découverte qui se rapporte à un nouveau procédé de stéréotype... »; 29 décembre 1820: «Vous m'obligerez infiniment de me donner quelques renseignements d'un Mr Guyet, éditeur du Constitutionnel qui s'est adressé à moi pour mon nouveau système de stéréotype qui a pour objet de multiplier les planches originales assez rapidement pour accélérer l'impression ou plutôt l'édition des feuilles quotidiennes. Si Mr Guyet (rue de Choiseul) est un personnage respectable, j'accéderai volontiers à ses propositions... ».

83 R. Beamish, Memoir.., op. cit., p. 343; H. Bagustt, The greatest Genius..., op. cit., p. 144.

84 Brunel Collection, Bristol, Journal de M. I. Brunel, mars 1822. Philip Taylor (1786-1870) et John Martineau s'étaient vraisemblablement installés à Londres, City Road, en 1820. Le premier né à Norwich avait pour frère Richard Taylor (17811858), imprimeur et éditeur scientifique chez qui travailla Frédéric Koenig, inventeur de la presse rotative. II semble que Taylor et Martineau aient reprit l'atelier que F. Koenig avait créé avec ses associés imprimeurs au centre de Londres à Clerkenwell (Whitecross Street) pour fabriquer ses presses et l'ait transformé en atelier d'équipement d'usines à gaz. L'imprimerie de Richard Taylor fut l'une des premières à être équipée de machine à vapeur ; "Note sur l'application de la machine à vapeur aux presses d'imprimerie », Bulletin de la Société d'Encouragement pour l'Industrie nationale, XVII, 1819, p. 69.

85 W. O. Henderson, J. C. Fisher and his diary of Industrial England, 1814-1851, 2005 ; P. Clements, Marc Isambard Brunel, op. cit., p. 114-116. 
pression, simple et pratique, connue en France pour avoir équipé l'usine à gaz comprimé des Ternes ${ }^{86}$. Celle-ci avait été construite par la société du gaz comprimé portatif que Charles Ternaux, le fils de Guillaume, avait créée en 1824 avec les associés de la London Portable Gaz Company, dont faisait partie John Taylor (1779-1863), le frère de Philip ${ }^{87}$. Ce dernier, rappelons-le, vint s'installer comme ingénieur en France à Paris en 1828, puis à partir de 1832 à Marseille M $^{8}$.

Durant ces quelques années, Marc Isambard commença à s'occuper de ponts, cette fois en relation directe avec son pays d'origine. Fin 1820, il reçut, en effet, une proposition pour construire à Rouen un pont sur la Seine, de part et d'autre de la pointe de l'île Lacroix en amont du fleuve ${ }^{89}$. II conçut deux projets, I'un en bois, l'autre en fer. Son offre ne fut malheureusement pas acceptée parce qu'il n'était pas rattaché, expliqua Quesnel son correspondant sur place, au Corps des Ponts-et-Chaussées ${ }^{90}$. La construction fut confiée, en effet, à Charles-François Mallet, ingénieur du Corps qui I'acheva en 1829. Une nouvelle proposition survint heureusement fin 1821, émanant cette fois du Ministère français de la Marine et des Colonies pour deux ponts à construire sur l'île de Bourbon - La Réunion - au dessus des rivières du Mât et

86 « Description d'une machine à vapeur à piston horizontal à haute pression et sans condensation de la force de dix chevaux construite par MM. Taylor et Martineau et en usage à I'usine des Ternes près Paris et dans plusieurs ateliers de France ", L'Industriel, III, 1827.

87 John Taylor avait mis au point auparavant un procédé de fabrication du gaz par distillation des huiles, concurrent du gaz hydrogène. "Description d'un appareil construit par M. Taylor pour l'éclairage du théâtre de Covent Garden à Londres », Bulletin de la Société d'Encouragement pour l'I'ndustrie nationale, XX, 1821, p. 208. Acte de société pour l'entreprise du gaz portatif : Arch. nat., Minutier Central des Notaires, Etude LXII, 852/B, 21 avril 1824 (capital de 480000 F, réparti en 160 actions).

88 Olivier Raveux, Marseille, ville des métaux et de la vapeur au XIXème siècle, Paris, CNRS Editions, 1998, p. 113 ; id., " Un technicien britannique en Europe méridionale, Philip Taylor (1786-1870) », Histoire, Economie et Sociétés, n²000 /2, Paris, 2000, p. 253.

89 R. Beamish, Memoir.., op. cit., p. 156. Archives de l'Académie des sciences, lettre de M. I. Brunel à Breguet fils: "J'ai reçu, il y a quelques jours, des nouvelles de Rouen. J'apprends que Mr Quesnel a reçu les dessins et mémoire... ».

90 Voir la lettre de Quesnel de novembre 1820: «Profitez de I'appui que peut vous accorder notre ambassadeur, pour ne pas vous présenter en France qu'après vous êtes attaché au Corps des Ingénieurs des Ponts et Chaussées, soit en qualité d'inspecteur, ou avec le rang d'ingénieur en chef. Autrement, je vous prédis que vous rencontrerez tant d'obstacles que rien ne vous réussira .... ». Cité par R. Beamish, Memoir.., op. cit., p. 156. de Saint-Suzanne, lesquels devaient pouvoir résister aux ouragans ${ }^{91}$. Isambard Kingdom fit le choix de ponts suspendus, I'un, celui de Sainte-Suzanne, à une travée de 131 pieds (40 m), l'autre, celui du Mât, à deux travées de la même longueur ${ }^{92}$. La mode des ponts suspendus démarrait alors en Angleterre, s'inspirant de l'exemple américain, et Isambard Kingdom venait justement de recevoir début octobre la visite de Claude Navier, en mission d'études sur le sujet pour le compte de l'administration des Ponts-et-Chaussées ${ }^{93}$. L'offre du Ministère, datée du 20 octobre, était relayée par le consul général à Londres, le baron Armand Louis Séguier ${ }^{94}$. Isambard Kingdom reçut manifestement aussi l'appui de Charles Debayssins, qui le premier avait installé dès 1815 des usines à sucre mécanisées, précisément sur les rivières du Mât et de Sainte-Suzanne ${ }^{95}$. Des dessins des deux ponts ayant été remis en avril $1822^{96}$, le contrat fut définitivement approuvé par le Ministère en juillet ${ }^{97}$. L'affaire prit cependant du retard, et c'est seulement courant 1823 que les éléments purent être progressivement fabriqués à Sheffield aux Milton Ironworks ${ }^{98}$. L'ouvrage, terminé fin avril, put y être examiné par I'Inspecteur général des Travaux maritimes, l'ingénieur des Ponts et Chaussées Joseph-Mathieu Sganzin (1750-1837). Navier eut aussi I'occasion de le voir en mai lors de sa deuxième mission d'études sur les ponts suspendus et en rapporta une description illustrée très précise. II apprécia, entre autres, I'emploi de fonte pour la charpente et le système de chaînes dites de re-

91 R. Beamish, Memoir..., op. cit., p. 178.

92 ॥ avait déjà conçu en 1818 le projet d'un grand pont suspendu sur la Neva à Saint-Pétersbourg, avec passage central réservé pour les grands navires.

93 Brunel Collection, Bristol. Lettre de M. I. Brunel à Breguet: «M. Navier a l'intention ensuite de faire une tournée vers l'Écosse pour y voir un pont de chaînes dont la portée est de 400 pieds. C'est un sujet qui ne peut manquer d'être très instructif et utile pour un ingénieur des Ponts-et-Chaussées ».

94 R. Beamish, Memoir..., op. cit., p. 180. On peut se demander si cette offre ne résulta pas directement de la visite de $C$. Navier. Le baron Séguier, très lié à la Société d'Encouragement pour I'industrie nationale, était en tout cas très actif dans les échanges techniques entre la France et l'Angleterre.

95 Brunel Collection, Journal de Marc Isambard, 2 juillet 1822 : Écrit à Mr Seguier et Mr Desbassyns concernant les ponts.

96 ld., Journal 22 mars: Mr Séguier communique les papiers relatifs à l'agrément du Ministère ; 18 avril : remise de rapport et dessins à Mr Seguier. M. I. Brunel effectuait parallèlement des tests de résistance.

97 R. Beamish, Memoir..., op. cit., p. 180. Ratification le 20 juillet pour un montant de 7000 livres, à verser dans les caisses des banquiers parisiens Mrs Outrequin et Jauge. M. I. Brunel avait à Paris un correspondant nommé Lees, qui traitait avec ces banquiers (cf. Journal de 1822).

98 P. Clements, Marc Isambard Brunel, op. cit., p. 79. 


\section{Les Brunel père et fils : deux célèbres ingénieurs anglais « Made in France »}

vers destiné à maintenir les planchers lors des ouragans ${ }^{99}$. Marc Isambard lui communiqua également à cette occasion les résultats d'essais de résistance du fer à la traction auxquels il s'était livré aux Milton Ironworks ${ }^{100}$. Les ponts furent ensuite expédiés à Londres en pièces détachées fin août, embarqués fin octobre et montés à la Réunion en décembre. Le Ministère de la Marine demanda alors à Marc Isambard une copie en grand des dessins des ponts, afin d'en faire des modèles à exposer dans le cabinet du Ministre. Fort de cette expérience, Marc Isambard reçut en novembre 1823 la visite de Marc Seguin venu en Angleterre s'informer sur le sujet en vue de la construction de son fameux pont de Tournon ${ }^{101}$. II revint voir Marc Isambard d'ailleurs avec son frère Paul fin 1825, cette fois à propos des machines à vapeur qu'ils souhaitaient installer sur leurs navires en construction destinés à la navigation à vapeur sur la Saône. Ils visitèrent aussi à cette occasion Taylor et Martineau auxquels ils commandèrent deux machines. Ce fut le point de départ des études qui allait conduire Marc Seguin à la mise au point en 1827 de la chaudière tubulaire (en collaborant, il est vrai, cette fois plutôt avec Georges Stephenson) ${ }^{102}$.

Élu fellow de la Royal Society en mai $1814^{103}$, Marc Isambard semble avoir assisté régulièrement aux séances. C'est là, en tout cas, qu'il entendit, en avril 1823, Michaël Faraday (1791-1867) faire part des découvertes qu'il venait de faire avec son maître Humphrey Davy (1778-1829) sur la liquéfaction à haute pression du gaz carbonique ou dioxyde de carbone (CO2) ${ }^{104}$. Des rencontres ultérieures avec Davy le persuadèrent rapidement de l'intérêt qu'il y aurait à se servir de la propriété qu'avait ce gaz liquéfié à se vaporiser en produisant une grande force mécanique

99 Claude-Louis-Marie-Henri Navier, Mémoire sur les ponts suspendus, 1823, p. 77 : « Ces ouvrages, concluait-il après avoir donné la description des ponts de Brunel alors en construction, qui se distinguent par la disposition ingénieuse des détails et par l'élégance de l'exécution, ont vivement intéressé le public et les artistes, et augmentent encore la réputation de cet habile ingénieur ».

$100 \mathrm{lbid}$., p. 248. Détermination des poids à partir desquels le fer « commence à s'étendre et se rompt». Brunel Collection, Bristol, Journal de Marc Isambard, 20 avril 1822, Mention de diverses expériences sur la déformation des chaînes.

101 Michel Cotte, Le fonds d'archives Seguin. Aux origines de la révolution industrielle en France 1790-1860, Privas, Archives départementales de l'Ardèche, 1997, p. 92.

102 Ibid., p. 83 et 91.

103 R. Beamish, Memoir.., op. cit., p. 140.

104 « Description d'un appareil dans lequel la vaporisation du gaz acide carbonique liquéfié produit un effet mécanique applicable à divers usages, inventé par M. Brunel, Bulletin de la Société d'Encouragement pour I'Industrie nationale, XXV, 1826, p. 76. pour faire fonctionner un nouveau type de moteur ${ }^{105}$. II y travailla sans succès y consacrant même d'importants moyens financiers pendant dix ans jusqu'en $1833^{106}$, prenant un brevet en juillet $1825^{107}$ qu'il chercha à breveter en France par l'intermédiaire des Ternaux ${ }^{108}$ (avec lesquels travaillaient alors Taylor et Martineau pour l'équipement de l'usine à gaz comprimé des Ternes). Ces efforts traduisaient chez lui un intérêt croissant porté aux questions physiques et chimiques, sans doute dû à la fréquentation de la Royal Society. C'est ce qu'atteste en tout cas la collaboration engagée dès avril 1825 avec Louis-Joseph Gay-Lussac (1778-1850) pour breveter en Angleterre un procédé servant à la fabrication de bougies par raffinage de suif, autrement dit de bougies stéariques dont le chimiste français venait de mettre au point le principe avec Eugène Chevreul (1786-1889) ${ }^{109}$. À partir de 1823, et surtout du 2 mars 1825, date du début du chantier, Marc Isambard se trouva absorbé de façon de plus en plus exclusive par sa dernière grande œuvre, la construction à Londres d'un tunnel sous la Tamise ${ }^{110}$. Elle dura près de 20 ans jusqu'à l'inauguration du 25 mars 1843, avec une interruption de sept ans due à une très grave inondation survenue le 12 janvier 1828. De ce remarquable chantier, on retiendra d'abord la mise en œuvre du fameux bouclier, châssis mobile comportant 36 cellules dans lequel les ouvriers pouvaient travailler à l'abri, et qui progressait au fur et à mesure de l'avancement du travail. Il avait fait l'objet d'un brevet pris dès janvier 1818 alors que Marc Isambard travaillait à son projet de fran-

105 Les circonstances sont racontées par S. Brindle, The man who built the world, op. cit., p 37. Cite le journal de M. I. Brunel, 30 mai 1823 : «Met sir Henry Davy who adverted to the discovery of a carbonic gaz to be used as a power... ". 106 II aurait dépensé 15000 livres, plus 200 livres de subvention de I'Amirauté. S. Brindle, The man who built the world, op. cit., p. 38. 10716 juillet 1825 : Certain mechanical arrangement for obtaining power from certain fluids, and for applying the same to various useful purpose ; H. Bagust, The greatest Genius., op. cit., p. 147. 108 Michel Cotte, De l'espionnage à la veille technologique, Belfort, Presses universitaires de Franche-Comté, 2005, p. 141. Échanges sur le sujet avec Ternaux en février 1826 et en août 1828 mentionnés dans les Journaux de M. I. Brunel conservés dans les archives de l'Institution of the Civil Engineers à Londres. Brevet pris le 5 mai 1826 par Andrieux pour Ternaux d'une " Machine destinée à être mise en mouvement par certain gaz en remplacement de la vapeur d'eau », L. M. Lohmüller, Guillaume Ternaux (1763-1833)..., op. cit., p. 446.

109 P. Clements, Marc Isambard Brunel, op. cit., p. 82. M. Cotte, De l'espionnage..., op. cit., p. 145. D'après les Journaux de M. I. Brunel, correspondance avec L. J. Gay-Lussac en avril et décembre 1825 pour une prise de brevet non seulement en Angleterre mais aussi en Amérique.

110 R. Beamish, Memoir..., op. cit., p. 202 (première description précise des étapes du chantier à partir de journal de M. I. Brunel). 
chissement de la Néva ${ }^{111}$. Mais l'ouvrage, on s'en doute, suscita de nombreuses autres innovations, entre autres dans le domaine des matériaux de construction, notamment pour la réalisation des parois du tunnel faites en briques dures jointes par un ciment dit romain, ciment naturel à prise rapide même en milieu humide, découvert par James Parker en 1796. Le chantier ayant commencé par le fonçage à Rotherhithe d'un grand puits de $16 \mathrm{~m}$ de diamètre et 20 de profondeur, son assèchement requit, on l'a vu, l'emploi de pompes et machines à vapeur modernes spécifiques, fournies par Brunel lui-même (machine à cylindres en $V$ renversé) et par Taylor et Martineau. En 1833, avant que les travaux ne reprennent, Marc Isambard procéda encore sur l'espace entourant le puits de Rotherthithe à l'édification d'un petit pont surbaissé en briques et pierres hourdés de ciment, construit à titre d'essai sans cintres ni échafaudages en liant simplement les lits de maçonnerie avec des bandes de fer et des lattis de bois. La technique convenait particulièrement pour construire, sans perturber la navigation, de petits ponts au-dessus des canaux ${ }^{112}$. Ce chantier offrit ainsi l'occasion à Marc Isambard de faire valoir pleinement jusqu'à la fin de sa carrière sa polyvalence et d'être perçu en France comme le modèle accompli de l'ingénieur civil.

\section{L'éducation d'Isambard Kingdom}

Isambard Kingdom naquit à Portsmouth le 9 avril 1806. De nationalité anglaise, il vécut d'abord à proximité de I'arsenal puis à Chelsea dès 1807. C'est son père qui fit sa première éducation, en français, ce qui fait que selon son compatriote le banquier Edward Blount (18091905), promoteur entre autres dans les années 1840 de la ligne Paris-Rouen-Le Havre, il parlait anglais avec un certain accent ${ }^{113}$. Très vite, il manifesta de l'intérêt pour

111 H. Bagust, The greatest Genius..., op. cit., p. 138.

112 «Construction des arches de ponts en briques et ciment hydraulique, sans cintres, ni échafauds, par M Brunel à Londres ", Bulletin de la Société d'Encouragement pour l'Industrie nationale, XXXIV, 1835, p. 523 (à partir d'informations communiquées par l'inspecteur des Ponts et Chaussées Mallet, qui les tenaient de M. I. Brunel lui-même). Rohault, « Voûtes en ciment hydraulique », Annales des Ponts et Chaussées, 1835, 1, p. 78 (cité par André Guillerme, Bâtir la ville, Révolutions industrielles dans les matériaux de construction. France-Grande Bretagne (1760-1840), Champ Vallon, 1995, p. 197). Le Bulletin de la Société d'Encouragement fit encore état en 1840 d'un procédé d'assemblage de pièces de charpente par des clefs en fer noyés dans l'asphalte, mis au point sur les chantier du tunnel (ibid., XXXIX, 1840, p. 348.).

113 Memoirs of Sir Edward Blount, 1902, p. 106 : « Amongst the engineers whit whom I was acquainted was the famous Isambard Brunel. He was of French descent, and, although born at Portsmouth, spoke English with a certain accent, owing to les sciences. « II n'aime pas beaucoup les livres excepté en mathématiques, pour lesquels il a du goût. II a commencé Euclide ... » écrivait son père en $1817^{114}$. Dès septembre 1814, celui-ci l'emmena à Hacqueville ; il y retourna en septembre 1817, confié aux bons soins de son grand cousin Brunel ${ }^{115}$. En 1820, alors que Marc Isambard n'excluait toujours pas de rentrer en France, et en tout cas pensait pouvoir y développer ses affaires, il décida d'envoyer son jeune fils y parfaire ses études. Isambard Kingdom y resta en tout deux ans et demi, d'avril 1820 à août 1822. Malheureusement les données dont on dispose sur ce séjour restent imprécises et même en partie contradictoires. Tous les biographes anglais répètent qu'il aurait d'abord été envoyé au collège de Caen, ce qui est possible, mais n'a été confirmé jusqu'alors par aucun document original ${ }^{116}$. II vint, en tout cas, s'installer à Paris en novembre 1820, Quai de l'Horloge, chez les Breguet (accompagné apparemment par sa mère alors que son père était retenu par ses affaires à Londres) ${ }^{117}$. " Ce n'est ni le grec, ni le latin qui devraient faire la principale partie de son éducation. La nature l'a doué de dispositions qui l'entraînent vers des connaissances plus utiles. On parle beaucoup du latin et du grec, et après tout les 19/20ème le

having passed the earlier years of his life in Paris, where he was educated at the Lycée Henri IV ».

114 Brunel Collection, Bristol. Lettre de M. I. Brunel du 2 septembre 1817 à son neveu.

$115 \mathrm{lbid}$., "Je confie mon petit garçon à vos soins. Comme il lui faut un mentor, je ne crois pas mieux choisir qu'en vous priant de modérer l'impétuosité de sa jeunesse ... Employez-le autant que vous le pourrez ... II ne faut pas le laisser prendre un exercice immodéré, il lui arrive souvent de s'épuiser... »

116 Par exemple, A. Vaughan, Brunel. Engineering KnightErrant..., op. cit., p. 7.

117 Brunel Collection, Bristol. Lettre du 28 novembre 1820 de M. I. Brunel à Breguet fils: "Vous m'aviez déjà donné des preuves (de vos bonnes dispositions à son égard) dans notre séjour à Paris il y a trois ans, lorsqu'il était petit écolier ... Comme dessinateur, il m'en remplacerait un de premier rang, non seulement par l'exactitude avec laquelle il copie ou il rend d'après une esquisse, mais encore avec beaucoup d'expédition. Ses dispositions sont rares et il aime le travail particulièrement celui qui fixe son attention et emploie ses mains. II ne peut trouver à mieux s'occuper qu'auprès de vous ». On ignore quand et dans quelle circonstance Marc Isambard avait fait connaissance des Breguet, Abraham Louis (1747-1823) et son fis Louis Antoine (1776-1858). II est possible que ce soit par l'intermédiaire de son beau-frère Thomas Mudge Jr., fils du grand horloger britannique Thomas Mudge (1717-1794), inventeur, entre autres, de l'échappement libre à ancre. T. Mudge Jr., lui-même apparem-ment aussi horloger, avait épousé Elisabeth Kingdom, sœur de Sophie, l'épouse de Marc Isambard. Les Breguet père et fils avaient fait plusieurs séjours à Londres entre 1792 et 1795, Louis Antoine avait même fait une partie de ses études en Angleterre. H. Bagust, The greatest Genius..., op. cit., p. 15. 


\section{Les Brunel père et fils : deux célèbres ingénieurs anglais « Made in France "}

perdent de vue, tandis que les langues vivantes peuvent tout nous dire et nous mettre à même de tirer parti de tous nos talents. Qu'il soit bon mathématicien, qu'il ait des connaissances chimiques, qu'il soit grand dessinateur : il sera initié à toutes les connaissances utiles et il pourra même s'y distinguer ... » écrivait encore Marc Isambard en guise de programme dans une lettre du 29 décembre 1820 à Breguet fils. Isambard Kingdom fut alors placé dans un établissement privé, I'Institution Massin, rue des Minimes, qui fonctionnait en partenariat avec le Collège Charlemagne ${ }^{118}$. On dispose de son relevé de notes pour le second semestre 1821. Marc Isambard qui apprenait I'Allemand, eut droit à l'appréciation générale suivante ${ }^{119}$ :

«Conduite irréprochable. Ce jeune homme a donné à tous ses professeurs la satisfaction la plus complète et il donne pour l'avenir les meilleures espérances », et par matières aux notations suivantes :

Devoirs religieux : «Remplis convenablement »

Français : " S'est perfectionné »

Latin : "S'en occupera autant que cela sera possible » Histoire, Géographie : «Bien »

Mathématiques, Physique, Dessin : «Très bien ».

$\mathrm{Au}$ vu de ces excellents résultats, Marc Isambard eut une nouvelle idée: "Notre intention serait de le faire entrer à l'École polytechnique, il s'agirait de le bien préparer» annonça-t-il à Breguet le 5 octobre $1821^{120}$. Conseil fut alors demandé à Claude Navier de passage, comme on l'a vu, durant sa mission sur les ponts suspendus : «Mr Navier doit être de retour à Paris. Il nous a promis de nous faire part du degré de force de notre jeune homme à l'égard de ses prétentions à l'École polytechnique... ». En février, la chose paraissait, en tout cas, entendue : « II y a tout lieu d'espérer son admission à l'École polytechnique au mois d'août prochain, pourvu toutefois qu'il ne se relâche pas... » ${ }^{121}$. A-t-il, comme

118 Elle avait été fondée en 1811 par Jean Massin, ancien préfet des études du collège Sainte-Barbe, qui l'avait installée dans I'ancien couvent des Minimes. Elle accueillait en 1820 180 pensionnaires et comptait parmi ses répétiteurs Adolphe Blanqui (1798-1854), futur professeur d'Économie industrielle au Conservatoire des Arts et Métiers et frère du révolutionnaire républicain Auguste Blanqui (1805-1881) qui y fut d'ailleurs élève. AN AJ/16/120.

119 Catalogue de vente de lettres autographes, Christie-South Kensington, 1996, n 25 (copie à la bibliothèque de I'Université de Bristol).

120 Brunel Collection, Bristol. II ajoutait: «Quels sont les moyens de l'y faire admettre. Vous m'obligeriez infiniment de me faire part de vos idées sur mon projet et quel serait le meilleur moyen pour le réaliser... ».

$121 \mathrm{lbid}$., lettre du 19 février 1822 de M. I. Brunel à M. Breguet. Dans cette lettre, il est question aussi d'un certain Francoeur, l'écrivent les biographes, suivi alors la classe préparatoires du lycée Henri IV ${ }^{122}$ ? A-t-il vraiment passé le concours d'entrée à Polytechnique ? Ou bien comme le rapportent de nombreux auteurs, n'a-t-il pas pu concourir parce qu'il était de nationalité britannique ${ }^{123}$ ? Ou encore, ce qui est le plus vraisemblable, a-t-il tout simplement échoué ? Le fait est qu'en août, il était à Calais et s'embarquait pour Douvres afin de rejoindre son père auprès duquel il allait bientôt reprendre du travail ${ }^{124}$. Jusqu'au grave accident de janvier 1828 où il faillit se noyer et qui allait nécessiter une convalescence de plusieurs mois, il collabora ainsi avec son père et poursuivit de ce fait sa formation en profitant de son expérience, travaillant d'abord sur le projet de moteur au gaz carbonique, puis de plus en plus à partir de 1825, au chantier de la Tamise. Bien qu'il n'ait pas suivi les cours de l'École polytechnique, Isambard Kingdom était passé en France dans le moule d'une école préparatoire et avait acquis non seulement une solide formation mathématique, mais aussi le goût de la performance technique et une façon de penser et se comporter qui le rapprochait des ingénieurs français. Durant son séjour à Paris, hébergé chez les Breguet, il avait eu aussi, malgré tout, I'occasion à ses heures perdues de tempérer un penchant peutêtre alors trop exclusif pour la théorie en se livrant à des exercices plus pratiques dans leurs ateliers d'horloger et de confectionner ainsi lui-même un graphomètre et un

probablement Louis Benjamin (1773-1849), répétiteur de mécanique à l'École polytechnique et enseignant au lycée Charlemagne (sans doute aussi à I'Institution Massin): «J'ai à vous prier d'être mon interprète auprès de Mr Francoeur et de lui exprimer combien je suis sensible à ses attentions pour mon jeune élève... »

122 À commencer par son fils. I. Brunel, The life of Isambard Kingdom Brunel..., op. cit., 1870, p. 15. Celui-ci, s'il indique bien que son père a fréquenté le Collège Henri IV, ne parle pas, en revanche, de l'épisode de l'École polytechnique. Concernant le séjour parisien de son père, il précise qu'il était d'abord destiné à lui apprendre le français et les mathématiques, et que sa vie durant, celui-ci conserva une grande admiration pour la méthode suivie en France pour les enseigner. II employait, ajoute-t il, son temps libre à examiner les différents travaux d'ingénieurs en cours alors à Paris et à en envoyer des descriptions et des dessins à son père.

123 A. Buchanan, Brunel..., op. cit., p. 18. II ajoute que Marc Isambard aurait encouragé son fils à prolonger son séjour « pour enrichir et mûrir son esprit ».

124 Brunel Collection, Bristol. Catalogue de vente de lettres autographes, Christie-South-Kensigton, 1996, n²6. Brunel Collection, Bristol. Lettre $d^{\prime} I$. K. à son père, 31 juillet 1822 : «I should receive for the third time two letters by the same post; one to tell me to go to Rouen, and the one to come home... »; Journal de M. I. Brunel, 21 août 1822 : « Isambard and Amédée arrived from France ». 
réveil matin ${ }^{125}$. De ce séjour à Paris, il conserva aussi un ami fidèle, Adolphe d'Eichtal (1805-1895) ${ }^{126}$, frère du saint-simonien Gustave, qu'il connut vraisemblablement au lycée Henri IV où il préparait l'École polytechnique au concours duquel, du reste, il échoua également. Plus tard après avoir voyagé en Angleterre et rendu visite à Isambard Kingdom durant sa convalescence ${ }^{127}$, Adolphe d'Eichtal s'installera comme banquier à Paris en 1831 et deviendra le premier président de la Compagnie du Paris Saint-Germain et I'un des principaux soutiens des frères Pereire dans leurs entreprises bancaires et industrielles.

\section{Une œuvre exemplaire}

Le premier chantier dont Isambard Kingdom eut à s'occuper personnellement fut celui du désormais célèbre pont de Clifton au-dessus des gorges d'Avon en aval de Bristol. La construction de ce pont suspendu pour lequel il déposa un premier projet en novembre 1829 eut une importance considérable pour l'avenir du jeune ingénieur de 23 ans. Ce fut le premier où il n'agissait plus comme simple adjoint de son père, et l'occasion aussi pour lui de faire ses premiers pas à Bristol, deuxième ville d'Angleterre un peu en déclin, il est vrai, face à la concurrence que lui menait Liverpool pour le commerce atlantique $^{128}$. Le concours fit cependant l'objet de péripéties. Isambard Kingdom présenta fin 1829 quatre projets alternatifs avec une portée du tablier suspendu central variant entre 700 et 1100 pieds (210 à 330 m), ce qui en faisait le plus long jamais construit ${ }^{129}$. Cette taille ayant suscité la méfiance du vieux Thomas Telford (1757-1834), constructeur en 1826 du pont de Menai (600 pieds), qui avait été appelé à donner son avis, un nouveau concours fut organisé en 1831, où T. Telford fut cette fois également candidat. Mais c'est le nouveau projet du jeune I. K. Brunel (630 pieds, soit $190 \mathrm{~m}$ de portée) qui fut finalement retenu après examen d'une commission où siégeait entre autres le mathématicien Davies Gilbert (1757-1834), alors président de la Royal Society ${ }^{130}$. Même si Isambard King-

125 Brunel Collection, Bristol. Lettre du 21 octobre 1821 à Breguet: " Il a mis en pratique la géométrie à l'aide du graphomètre qu'il a fabriqué chez vous, sans faire le moindre tort aux artistes du Quay de l'Horloge... »; lettre du 22 février 1822 à Breguet : " II nous a fait part de son réveil matin fait chez Mr Breguet. II est vraiment heureux de se trouver ainsi dans son ancien élément... ».

126 A. Buchanan, Brunel, op. cit., p. 30. Nicolas Stoskopf, Banquiers et financiers parisiens, Paris, Picard/Cénomane, 2002, p. 158.

127 A. Buchanan, Brunel, op. cit., p. 30.

128 Ibid., (chap. 4 : « Bristol »).

129 Michael Pascoe, Adrian Andrews, "Suspensia Vix Via Fit. The saga of the building of the Clifton suspension bridge », dans A. Kelly, M. Kelly éd., Brunel. In love whith impossible, op. cit., p. 85. 130 Alfred Pugsley, «Clifton Suspension Bridge », dans A. Pugsley dom fut bel et bien le concepteur du projet, on sait que son père y participa activement, ayant lui-même conçu et construit en 1822-1823 les deux ponts suspendus et l'île de La Réunion et eu alors largement l'occasion, comme on l'a vu, de discuter de la question y compris sur le plan théorique avec Marc Séguin et Claude Navier ${ }^{131}$. Dans les débats qui eurent lieu à Bristol en 1831, et qui portèrent entre autres sur le choix de chaînes au lieu de câbles, Isambard Kingdom ne se priva d'ailleurs pas de faire valoir qu'il connaissait intimement les frères Seguin ${ }^{132}$. La construction du pont de Clifton connut malheureusement de sérieuses difficultés, principalement d'ordre financier. A peine commencé en août 1831, le chantier fut arrêté. ॥ reprit en 1836, mais s'arrêta à nouveau en 1843, et le pont ne fut finalement achevé qu'en 1864, cinq ans après la disparition d'Isambard Kingdom et en utilisant, du reste, les chaînes d'un autre pont suspendu construit par lui en 1845, le pont pour piétons de Hagerford situé à Londres sur la Tamise et démoli dès $1860^{133}$.

Après I'affaire du pont de Clifton, Isambard Kingdom se trouva à partir de 1833 successivement en charge de la modernisation du port de Bristol, de la construction du navire transatlantique Great Western et de la création de la ligne de chemin de fer Londres-Bristol (Great Western Railway - GWR). II devint dès lors complètement absorbé par ces travaux, y déployant une énergie considérable, une aptitude étonnante à mener de front des entreprises relativement différentes, tout en conservant une exceptionnelle inventivité. Cette activité intense eut visiblement pour conséquence un certain détachement de la France, si bien qu'Isambard Kingdom n'eut pas, contrairement à d'autres ingénieurs anglais contemporains (Joseph Locke entre autres et même les Stephenson pour la fourniture de locomotives), d'engagements contractuels avec des entreprises ou l'Etat français. Un examen attentif de ce que se passa alors dans les années 1830-1850 de part et d'autre de la Manche montre cependant que les ponts furent loin d'être coupés; il suggère au contraire l'existence de contacts quasi-permanents, fondés sur des voyages et des visites, des échanges épistolaires, ou simplement un suivi régulier de ce qui pouvait être écrit dans une presse générale et spécialisée de plus en plus abon-

éd., The Works of Isambard Kingdom Brunel. An engineering Appreciation, Londres, Institution of Civil Engineers, 1976, p. 51.

131 À la vue de dessins du pont suspendu des Invalides construit à Paris par C. Navier entre 1824 et 1826, Marc Isambard se serait, d'ailleurs, montré très sceptique . L.T.C. Rolt, Isambard Kingdom Brunel, op. cit., p. 26.

132 M. Pascoe, A. Andrews, op. cit., p. 99. «In a forthright letter he stated that he had long studied the use of wire and was intimate with the principal French manufacturers, Mrs Seguin ». 133 « Hungerford bridge », dans M. Kelly, M. Kelly, op. cit., p. 110. 


\section{Les Brunel père et fils : deux célèbres ingénieurs anglais « Made in France »}

dante sur les questions techniques, notamment ferroviaires. En se référant aux nombreuses études anglaises effectuées depuis une trentaine d'années sur les différents chantiers d'Isambard Kingdom durant cette période, il est même possible d'établir un parallèle avec ce que I'on peut savoir des travaux réalisés au même moment en France alors que démarrait la construction ferroviaire et que naissait le métier d'ingénieur civil à l'anglaise. Compte tenu du caractère encore une fois informel de ces échanges, un bilan n'est cependant envisageable qu'en s'appuyant sur quelques exemples concrets et en s'efforçant de repérer des simultanéités et des similitudes, pas forcément du reste avouées. Là où la relation a été manifestement la plus suivie, c'est avec les entreprises réalisées dans le cadre d'abord de la compagnie du Paris - Saint-Germain, ensuite du Crédit Mobilier, où rappelons-le, Adolphe d'Eichtal, I'ami personnel d'Isambard Kingdom, occupa longtemps à côté des frères Pereire une position de premier plan. Or dans ces affaires, intervenait généralement comme ingénieur, Eugène Flachat (1802-1873), sympathisant saint-simonien comme les Pereire et les d'Eichtal, considéré comme le père de l'ingénierie française $^{134}$. Flachat avait fait connaissance des Brunel, père et fils, dès 1826 lorsqu'il était venu visiter le chantier du tunnel sous la Tamise ${ }^{135}$ tandis qu'il s'occupait avec son demi frère Stéphane Mony de l'étude d'un canal entre Paris et Rouen ${ }^{136}$. En 1827, il était encore en correspondance avec Marc Isambard qui lui fournit un plan de docks ${ }^{137}$ (qu'il utilisa peut-être en 1833 lorsque de retour d'un séjour à Odessa, il travailla à la construction des docks dits du Marais à Paris sur le canal Saint-Martin).

La première affaire pour lesquels Brunel et Flachat collaborèrent de façon attestée, fut l'installation d'un chemin de fer à mode de propulsion originale - chemin de fer dit atmosphérique - dont ils s'occupèrent comme ingénieurs en chef pratiquement au même moment, entre 1844 et 1847, I'un pour la ligne du Sud Devon (85 m entre Exeter et Plymouth), I'autre pour le prolongement et I'aboutissement de la ligne du Paris - Saint-Germain depuis Le Pecq $\left(5 \mathrm{~km}\right.$ en forte pente) ${ }^{138}$. Ce système

134 Alain Auclair, Les ingénieurs et l'équipement de la France. Eugène Flachat (1802-1873), Mâcon, Écomusée du Creusot, 1999. 135 Eugène Flachat, " Isambard Brunel », Mémoires et Compte Rendu des Travaux de la Société des Ingénieurs civils, Paris, 1859, p. 410. "Quand nous I'avons connu, en 1826, il travaillait, sous la direction de son père, au percement du tunnel sous la Tamise ; il avait vingt ans $»$.

136 A. Auclair, Les ingénieurs et l'équipement de la France..., op. cit., p. 4. La société avait été constituée en février 1825.

137 M. Cotte, De l'espionnage..., op. cit., p. 145.

138 Paul Smith, Les chemins de fer atmosphériques, In Situ, n 10, 19 mai 2009 (revue en ligne). qui provoqua alors un véritable engouement comme alternative à l'utilisation de locomotives à vapeur, reposait sur l'obtention du vide dans un tube de fonte posée au milieu de la voie. II avait fait l'objet de nombreuses recherches expérimentales d'abord en Angleterre, mais aussi en France jusqu'à ce qu'une technique s'impose, celle mise au point par Samuel Clegg et les frères Jacob et Joseph Samuda. Leur technique, testée à Paris dès 1838 dans la grande entreprise mécanique de Chaillot, avait pu être mise en œuvre sur deux petites lignes, la première ouverte en mars 1844 en Irlande entre Kingstown et Dalkey près de Dublin $(2,9 \mathrm{~km})$, la seconde construite en 1844-1845 entre Londres et Croydon. L'avantage attendu était une économie de combustible, davantage de sécurité et de confort, la possibilité de gravir de fortes pentes, ce qui permettait de simplifier les tracés et d'éviter la construction de coûteux ouvrages d'art. Dès 1842, I. K. Brunel avait pensé équiper d'un tel système la ligne Milan-Gênes dont l'étude venait de lui être confiée ${ }^{139}$. Mais pour passer à la réalisation, il lui fallut attendre la construction d'un chemin de fer côtier le long de la Riviera anglaise dans le South Devon dont le principe fut acquis en juillet 1844. Du côté du Paris Saint-Germain, la décision fit suite à l'attribution en août 1844 d'une généreuse subvention de l'Etat qui souhaitait voir tester cette technique jugée révolutionnaire. Dans cette perspective, Émile Pereire, le directeur de la Compagnie, et son ingénieur Flachat firent à l'automne suivant un séjour d'un mois Outre Manche pour observer les lignes existantes ou en projet, et rencontrer entre autres Brunel ${ }^{140}$. Plusieurs lettres de celui-ci à son ami d'Eichtal, président de la Compagnie, évoquent aussi I'affaire ${ }^{141}$. On sait enfin que Brunel visita le chantier de Saint-Germain, alors bien avancé, en septembre 1846 en revenant $d^{\prime} \mid{ }^{\prime}$ alie ${ }^{142}$. Ces quelques informations ne disent

139 Journal des Chemins de Fer, 18 juin 1842: «M. Brunel, de retour de son voyage en Italie où il est allé étudier la voie de fer qu'on se propose d'établir entre Gênes, Milan et Turin, a I'intention d'appliquer le système atmosphérique à la partie de la ligne qui gravira les Apennins. Les chutes d'eau qu'on trouve en abondance dans ces montagnes seraient utilisées pour mettre en mouvement les pompes à air ».

140 A. G. extraordinaire de la Compagnie du Paris - SaintGermain, 8 novembre 1844

141 Brunel Collection, Bristol. Lettres à d'Eichtal des 20 juin 1843, 14 juin 1844, 27 décembre 1844, 2 janvier 1845, 4 août 1845. Elles y abordent toutes sortes de sujets ferroviaires. Dans celle du 27 décembre 1844, on lit: " What are you doing in the atmospheric ?».

142 Journal des Chemins de Fer, 12 septembre 1846. « M. Brunel a examiné lundi dernier, avec M. Samuda, qui se trouvait à Paris, les travaux du chemin de fer atmosphérique en construction à Saint-Germain; il est entré dans les plus grands détails. Ces 
évidemment pas tout. II y eut sûrement des échanges de plans et de documentation, sans doute une correspondance régulière, voire des visites sur place d'ingénieurs et de collaborateurs en charge des travaux (comme Ernest Deligny et Constantin de Regel côté français). Même si les deux ouvrages présentaient des différences significatives - une vraie ligne pour I. K. Brunel, à voie unique d'ailleurs, mais à fonctionnement atmosphérique dans les deux sens ; un simple prolongement pour celle de Flachat et fonctionnant par simple gravité à la descente, tout en comportant des ouvrages d'art (viaduc, tunnel) assez complexes - c'est la même technologie qui fut adoptée, avec le même enthousiasme. Les fournisseurs de matériel - grosses machines fixes de pompage, tuyaux de fonte... - ayant été, il est vrai, tous anglais pour le South Devon, français et belges pour le Saint-Germain, il n'y eut pas sur ce chapitre d'échanges possibles. Après I'ouverture de la ligne du Paris-Saint-Germain en avril 1847 et celle du South Devon en septembre suivant, les suites furent enfin carrément différentes. Très vite, on se rendit compte partout que le système atmosphérique n'était sans doute pas viable économiquement. Mais la conclusion fut immédiate en Angleterre, il fut abandonné sur le South Devon dès août 1848, et I'expérience resta comme I'un des plus sérieux échecs d'Isambard Kingdom. Au Paris-Saint-Germain, par contre, il continua à fonctionner jusqu'en 1860, il est vrai pour une simple bout de ligne et sans connaître de postérité.

Les choses se présentent un peu différemment pour la construction des ponts, un domaine pour lequel on ne dispose d'aucun témoignage d'échanges concrets, mais pour lequel on est en droit de supposer un intérêt attentif à tout ce qui se passait de part et d'autre de la Manche et une application rapide des leçons qui pouvaient être tirées. En tant qu'ingénieur du Great Western Railway, Isambard Kingdom eut à concevoir et construire de nombreux ponts et viaducs. Beaucoup furent construits en brique tel le fameux viaduc de Maidenhead, ouvrage à deux travées de $39 \mathrm{~m}$ et arcs très surbaissés achevé en 1838 pour lequel Isambard Kingdom bénéficia évidemment des constructions expérimentales réalisées par son père cinq ans auparavant sur le chantier de Rotherhithe ${ }^{143}$. En Cornouaille où le relief est accidenté, Brunel réalisa aussi entre 1850 et 1859 , par souci d'économie, une série de 43 viaducs en bois remarquables d'ingéniosité. Alors que Brunel était perçu comme très hostile à la fonte - ce qu'il proclamait d'ailleurs -, il a pu récemment être montré

messieurs ont adressé à M. Eugène Flachat leurs félicitations pour le soin et I'habileté qu'il a déployés dans l'exécution si délicate de ce bel appareil... ».

143 I. Brunel, The life..., op. cit, p. 133. J. B. B. Owen, «Arch bridges », dans A. Pugslet, The works..., op. cit., p. 89. que comme Robert Stephenson, il construisit sur la ligne Londres-Bristol à partir de 1837 un certain nombre de ponts en fonte ${ }^{144}$, dont l'un édifié en 1838 pour franchir à Londres le canal de Paddington, a été récemment retrouvé. Ce qui est également surprenant, c'est l'intérêt qu'il porta précocement à la construction en fer, ce avant ses deux célèbres réalisations, le pont de Chepstow sur la Wye au sud du Pays de Galles (1852) et le fameux pont Royal Albert à arc tubulaire destiné au franchissement de la Tamar à Saltasch en Cornouaille, conçu en 1855 et inauguré en mai $1859^{145}$. L'intérêt des Britanniques pour la construction en fer remonte aux conséquences de I'accident du pont sur la Die construit par R. Stephenson, survenu en mai 1847, et aux travaux qui suivirent d'Eaton Hogkinson (1789-1861) et William Fairbairn (1789-1874) sur les résistances comparées de la fonte et du fer, menés en vue de la construction, toujours par R. Stephenson, du célèbre pont Britannia en tôles rivetés, ouvert à la circulation en mars $1850^{146}$. Or Brunel, très attentif à ces travaux, se lança aussi dès 1847 dans la construction en fer. II rebâtit ainsi en fer l'ancien pont en fonte d'Uxbridge Road endommagé par le feu cette même année, et réalisa aussi en fer un pont sur un canal dans le port de Bristol et le pont sur la Tamise d'une petite ligne conduisant à Windsor, I'un et I'autre achevés dès $1849^{147}$. Cette construction en tôles ou fers plats supposait la disponibilité de la part de l'industrie sidérurgique de fers laminés plus ou moins profilés et la maîtrise de l'assemblage par rivets. Brunel les avait; la sidérurgie lui fournissait des rails, notamment ces rails en $U$ renversé qu'il avait mis au point dès 1836 (appelés «bridge rail»), et le rivetage pratiqué en chaudronnerie pour la fabrication de chaudières de machine à vapeur et la construction navale lui était familier. Le travail du fer était aussi familier

144 S. Brindle, The man who built the world, op. cit., p. 110. Le premier fut celui d'Uxbridge Road à Hanwell, West London, qui posa quelques problèmes à son concepteur. S. Brindle, M. Tucker, "First Cast Iron Bridges and the Uxbridge Road Fiasco", Transactions of the Newcomen Society, vol. 78, 2008, p. 25.

145 A. R. Kingdom, Brunel's Royal Albert Bridge, Newton Abbot, Ark Publications, 2006.

146 An account of the construction of the Britannia and Conway Bridges, 1849; W. Fairbairn, On the application of cast and wrought iron for the building purposes, New-York, 1854 (p. 192 : «Pendant plusieurs années, on a ignoré ou du moins on n'a connu que très imparfaitement la supériorité que présente le fer malléable pour la construction des poutres ... L'élasticité, la ductilité et la cohésion du fer sont restées presque complètement inconnues jusqu'à ce que ce sujet eût été étudié dans des applications en grand, lorsqu' on chercha les moyens de faire franchir les détroits de Conway et de Menai au chemin de fer de Chester à Holyhead... ".

147 S. Brindle, The man who built the world, op. cit., p. 113. 


\section{Les Brunel père et fils : deux célèbres ingénieurs anglais « Made in France »}

à Eugène Flachat qui avait eu l'occasion de moderniser un certain nombre d'usines sidérurgiques dans les années 1835-1840. Pour la couverture des halles de la gare SaintLazare, il avait également réalisé dans les années 1846-47 de grandes charpentes métalliques, combinant fonte et fer forgé selon le principe conçu par le jeune ingénieur centralien Camille Polonceau en $1838^{148}$. Un voyage en Angleterre en 1849 lui permit aussi de voir la plupart des grands ponts alors en construction ${ }^{149}$. II n'est donc pas étonnant que lorsqu'il s'agit après le coup d'arrêt apporté aux travaux ferroviaires par les évènements de 1848, de construire à nouveau des ponts, c'est en fer qu'ils furent réalisés. Les premiers furent les ponts de Clichy et d'Asnières (1851-1854), puis vinrent à partir de 1855 les trois ponts édifiés pour franchir la Garonne et la Dordogne par la Compagnie du Midi, une compagnie créée en 1852 par les Pereire dont Flachat fut nommé ingénieur en chef et qui se montra particulièrement innovante (elle adopta notamment le rail Brunel). La construction de tous ces ponts fut confiée à l'entreprise Gouin, entreprise de construction de locomotives fondée en 1845 par Ernest Gouin qui avait d'abord travaillé au service du matériel de la Compagnie du Paris-Saint-Germain. Pour ces ponts, Flachat et ses jeunes ingénieurs, en particulier Henri de Dion, s'inspirèrent, à l'évidence, de l'expérience anglaise en matière de construction en fer ${ }^{150}$, bien qu'ils fussent de conception plus simple - des ponts en tôle rivetées à poutre droite. Grâce à une méthode de calcul mise au point par Émile Clapeyron qui signa là d'une certaine façon la naissance de la résistance des matériaux, le pont $d^{\prime}$ Asnières, puis les ponts de la Compagnie du Midi furent préalablement calculés, leurs élément dessinés, ensuite préfabriqués en atelier et percés aux bons emplacements de telle sorte qu'ils puissent être montés et rivetés sur

148 Jean-François Belhoste, « La gare Saint-Lazare, témoin exceptionnel des débuts de la construction métallique en France », Revue d'Histoire des chemins de fer, $n^{\circ} 20-21,1999$, p. 21.

149 Mémoire et Compte rendu de la Société des Ingénieurs civils, 1849-4, p. 5. La note évoque les observations faites sur les « constructions des grands ponts de Menai, de Conway, de Newcastle... ».

150 Voici ce qu'écrivit plus tard Flachat sur la question : « Brunel a fondé, avec Stephenson, l'emploi du fer dans les travaux d'art ... On affirmait en France que les ingénieurs anglais n'ayant pas d'école, n'avaient pas de science ; et cela se disait au moment où Brunel fils et Stephenson élevaient, avec la matière qui peut être le plus hardiment et le plus sûrement soumise au calcul, des œuvres gigantesques que la science a sanctionnées et ... encore critiquées. Ces travaux, nous les connaissons tous, nous en avons avidement lu les descriptions; ou nous les avons visités, car nos pèlerinages d'étude se sont toujours accomplis, de préférence, dans le pays où ces hommes de génie ont montré la puissance de leurs conceptions... ». "Isambard Brunel », op. cit., p. 414. les chantiers avec le maximum d'efficacité. Deux jeunes ingénieurs employés par Flachat en tirèrent la matière d'un livre qui parut peu après avec la préface suivante: «L'introduction en France des ponts en tôle date de la construction des ponts de Clichy et $d^{\prime}$ Asnières ... Le pont $d^{\prime}$ Asnières est le premier pont important et construit sur des idées rationnelles que nous ayons possédé... ». Même si Flachat ne réalisa pas exclusivement des ouvrages en fer riveté, puisqu'il utilisa par exemple la brique et la fonte pour les ponts construits en 1855 sur la ligne Paris-Auteuil à l'ouest de Paris ${ }^{151}$, on peut considérer que ses réalisations du début des années 1850 marquèrent le début d'une construction métallique spécifiquement française, caractérisée par une grande maîtrise du dessin et du calcul, une préfabrication maximale et une grande attention aux techniques de montage ${ }^{152}$. Ce savoir-faire s'exportera d'ailleurs rapidement, en particulier vers les Empires russe et austro-hongrois, exploité par de grandes entreprises, telles la Société de Construction des Batignolles (ancienne entreprise Gouin), Le Creusot des Schneider ou l'entreprise de J. F. Cail, et d'autres nouvellement créées dont la plus réputée est celle que Gustave Eiffel fonda en 1865.

Les relations de Brunel avec la France concernant la construction navale sont, en l'état, encore moins documentées. Celui-ci s'étant principalement intéressé à la navigation à vapeur transocéanique, on peut comprendre que le domaine n'ayant pas encore été abordé par des entreprises de navigation françaises, il n'ait pas suscité beaucoup d'échanges. Les trois navires que Brunel réalisa furent cependant tous révolutionnaires: le premier, le Great Western construit à Bristol en 1836-1837 (en bois et muni de roues à pales) fut le premier navire à vapeur à assurer une liaison régulière entre l'Angleterre et les EtatsUnis; le second, le Great Britain, construit entre 1840 et 1843, fut le premier transatlantique construit en fer et équipé d'une hélice ${ }^{153}$; le troisième, le Great Eastern dont la construction fut entreprise à Londres en 1853 et qui ne fit sa première traversée transatlantique qu'en juin 1860, frappe par son gigantisme et toutes sortes d'innovations dont un mode de construction cellulaire,

151 Auguste Perdonnet, Traité élémentaire des chemins de fer, tome 1, 1855, p. 355.

152 Jean-François Belhoste, "Les centraliens et la construction métallique de 1830 à 1914, dans D. Barjot, J. Dureuil éd., 150 ans de génie civil : une histoire de Centraliens, Paris, Presses de l'Université Paris-Sorbonne, 2008, p. 59.

153 Ewan Corlett, The iron ship..., op. cit. La description qu'on y trouve, fondée sur l'examen du navire conservé à Bristol, fait comprendre le système d'assemblage par rivets utilisé par I. K. Brunel, et comment il a pu s'en inspirer peu après pour la construction de ses ponts en fer. 
à double coque, permettant d'éviter la propagation de l'eau et des incendies. Ceci dit la construction navale française n'était pas restée inactive. Elle s'était mise dès les années 1820, avec entre autres l'entreprise Manby et Wilson de Charenton, puis celle de François Cavé à Paris, à la construction de bateaux à vapeur en fer pour la navigation sur la Seine et transmanche. Dans le domaine militaire, la construction en 1857 du cuirassé La Gloire avait été fut plus tard une révolution. S'agissant de la propulsion par hélice, Brunel ne pouvait ignorer les travaux de Frédéric Sauvage (qui avait pour protecteur le Baron Séguier, fils de l'ancien consul à Londres et mécanicien, avec lequel il entretenait une correspondance) ${ }^{154}$; ni ce qu'entreprenait le constructeur du Havre Augustin Normand en association avec le mécanicien de Londres John Barnes qui aboutit au lancement en 1842 du premier navire à hélice français, Le Napoléon, même si dans le rapport qu'il écrivit en octobre 1840 pour justifier son choix ${ }^{155}$, il ne faisait référence qu'à L'Archimède (premier bateau à hélice anglais construit par Francis Pettit Smith et qui effectua son premier trajet en mai 1839). Quant à Flachat, il est évident qu'il suivait ces travaux, mais plutôt en spectateur attentif, prenant notamment le temps lors de son voyage en Angleterre de 1849 de les observer parmi d'autres choses ${ }^{156}$. Et c'est finalement lui qui fit connaître par son ouvrage en 1865, les caractéristiques précises des trois bateaux de Brunel et spécialement du Great Eastern - Le Léviathan -, auquel il consacra un long chapitre ${ }^{157}$. II I'avait d'ailleurs vu en construction à Londres ayant été convié par Isambard Kingdom à sa première tentative de lancement en $1857^{158}$. Lorsqu'il fit paraître son livre, Flachat était devenu avec son ami Emile Clapeyron, membre du Conseil d'Administration de la Compagnie Générale Transatlantique, encore une entreprise des Pereire, et à ce titre chargé d'étudier les meilleures conditions d'exploitation d'une ligne entre Le Havre et New-York dont cette nouvelle compagnie était devenue concessionnaire exclusif ${ }^{159}$. II fit pour cela en 1861 un nouveau voyage en Grande-Bretagne pour visiter les principaux chantiers navals, notamment ceux de la Tamise et des environs de Glasgow. L'étude aboutit à I'installation en 1861 des chantiers à Saint-Nazaire, créée par la Compagnie Transatlantique en association

154 Brunel Collection, Bristol.

155 E. Corlett, op. cit., p. 56.

156 Bulletin de la Société des Ingénieurs civils, 1849-4, p. 5.

157 Eugène Flachat, Navigation à vapeur transocéanienne. Etudes scientifiques. Etudes économiques et de statistique, Paris, 1866 (3 vol.).

158 Brunel Collection, Bristol. Lettre du 17 novembre 1857.

159 AN F/12/6777. Crée au capital de 40000 millions F, elle prenait la suite de Compagnie Générale Maritime fondée en 1854. avec l'écossais John Scoot des chantiers de Greenock, où furent construits dés 1865 les premiers navires français transatlantiques à hélice, le Ville de Paris et le Pereire, sur le modèle mais en nettement moins grand du Great Eastern.

Sauf preuve du contraire, il ne semble pas avoir existé de collaboration concrète entre Brunel et les entreprises animées par les Pereire, d'Eichtal et Flachat. Son influence s'est fait sentir sans doute uniquement de façon informelle, en particulier via l'exemple qu'il offrit dans l'exercice d'un nouveau métier, celui d'ingénieur civil, pratiqué au sein de cabinets indépendants, fonctionnant un peu sur le modèle du cabinet d'architecte, dans le cadre d'un nouveau domaine, l'ingénierie. À partir du moment, où il devint en 1833 ingénieur du Great Western Railway, Isambard Kingdom combina, en effet, deux activités, celle d'ingénieur appointé et celle d'animateur d'une équipe d'ingénieurs réalisant des études pour les chantiers en cours, ferroviaires et autres, et élaborant des projets pour de nouveaux clients ${ }^{160}$. Ce cabinet semble s'être surtout développé à partir de 1836 lorsqu'Isambard Kingdom eut emménagé au centre de Londres, 18 Duke Street, ayant son habitation, du reste, au dessous de son bureau. Vers 1850, il comptait entre 30 et 40 personnes, à une époque, il est vrai, où les affaires allaient très bien et où se préparaient de gros projets pour I'Inde (Bengale) et l'Australie (Melbourne). L'organisation de ce cabinet était particulièrement rigoureuse, I. K. Brunel dirigeait son équipe d'une main ferme, avec beaucoup d'exigence. Or coïncidence curieuse, Flachat mit en place à peu près au même moment, en fait dès 1833, une organisation du même genre dans lequel travaillaient de jeunes ingénieurs pour la plupart sortis de l'École Centrale des Arts et Manufactures, nouvellement créée ${ }^{161}$. Dans un

160 A. Buchanan, Brunel, op. cit., p. 153 (chap. 10: "The professional Man »).

161 A. Auclair, Les ingénieurs et l'équipement de la France..., op. cit., p. 109. Voici comment Flachat relata les choses: « Le puissant concours de Petiet permit bientôt un développement considérable des travaux d'un groupe formé de jeunes ingénieurs tous sortis de l'École centrale, et dont la place s'est marquée, depuis au premier rang, dans la profession du génie civil. Le domaine des applications du génie civil était alors fermé par l'administration publique, à l'industrie et aux ingénieurs. La construction des canaux était aux mains de l'Etat. II en était de même des ports; les docks étaient vivement disputés... Restaient les applications dans I'industrie. La fabrication du fer commençait une transformation qui réclamait le concours des ingénieurs; les ateliers de construction, l'éclairage des villes par le gaz, les distributions d'eau s'offraient presque seules à l'activité de cette nouvelle profession... »: Eugène Flachat, " Notice nécrologique sur Jules Petiet », Mémoires de la Société des Ingénieurs Civils, Paris, 1872. 


\section{Les Brunel père et fils : deux célèbres ingénieurs anglais « Made in France »}

premier temps, avec le concours notamment de Jules Petiet (1813-1871) ${ }^{162}$, le cabinet Flachat se spécialisa dans la modernisation d'usines sidérurgiques (Le Creusot, Vierzon, Abainville...), le tracé de canaux et la construction de docks, et c'est seulement à partir de 1842 lorsque Flachat eut obtenu le poste d'ingénieur en chef de la Compagnie du Paris-Saint-Germain, qu'il consacra l'essentiel de son activité aux questions ferroviaires, un peu comme faisait Brunel Outre-Manche. De nouveaux collaborateurs furent recrutés dont certains comme Henri de Dion et Léon Molinos firent ultérieurement de grandes carrières dans le génie civil et l'industrie. Ce qui différencia alors la France et l'Angleterre en la matière, c'est l'existence d'une école spécialement consacrée à la formation d'ingénieurs civils (mêlant sciemment enseignement théorique et pratique) alors qu'OutreManche, cette formation continuait à se faire sur le tas. II en sortit des ingénieurs particulièrement experts en génie civil, spécialement en matière de construction métallique, dont la compétence allait largement s'exporter durant la deuxième moitié $d u X X^{e}$ siècle $^{163}$. Notons qu'au moins deux élèves de l'École Centrale travaillèrent au milieu des années 1840 chez Brunel, Arthur Corneck et César Zanolini, italien, né à Bologne, qui dirigea ultérieurement la grande manufacture d'armes de Terni, près de Rome ${ }^{164}$.

\section{Postérité}

Comme on l'a déjà souligné au début de cet article, les Brunel père et fils sont un peu oubliés en France de nos jours. Au XIXe siècle, ils y furent cependant reconnus et honorés comme anciens compatriotes, de façon différente du reste, ce qui se comprend puisque leurs relations avec leur pays d'origine ne furent pas comparables. Après I'arrêt du chantier de la Tamise en 1828, Marc qui avait alors 60 ans pensa à nouveau entreprendre quelque chose en France. II y séjourna longuement fin 1829 et fit sans succès deux propositions l'une pour une entreprise d'adduction d'eau à Paris, qui reprenait une projet déjà formulé en 1817, I'autre pour la construction d'une ligne ferroviaire entre Paris et Rouen ${ }^{165}$, en se présentant

162 Jean-Pierre Willot, Jules Petiet (1813-1871). Un grand ingénieur du XIXe siècle, Paris, 2006 (hors commerce).

163 J.-F. Belhoste, «Les centraliens et la construction métallique... », op. cit.

164 D'après les annuaires de l'époque (archives de l'École Centrale). 165 On trouve évoqués ces deux chantiers qui faisaient alors l'objet de débats et de diverses propositions dans l'ouvrage que publia en 1832, Eugène Flachat avec son frère Stéphane et ses amis Clapeyron et Lamé. Émile Clapeyron, Gabriel Lamé, Eugène Flachat, Stéphane Flachat, Vues politiques et pratiques sur les travaux publics en France, Paris, 1832. Un projet de chemin de fer entre Paris et Le Havre avait été proposé dès 1826 dans les deux cas comme le correspondant d'entreprises à capitaux anglais ${ }^{166}$. II vint aussi en Normandie, fit à I'Académie des Sciences, Belles Lettres et Arts de Rouen une conférence très remarquée sur le chantier de la Tamise ${ }^{167}$, reçut à cette occasion la légion d'honneur des mains du préfet, et séjourna à Hacqueville chez son frère ${ }^{168}$ qui l'emmena entre autres choses visiter la grande entreprise voisine de cuivre et zinc de Romilly sur Andelle. « Venu en 1829 en France pour voir encore une fois sa famille, raconta le directeur Lebrun, Brunel voulut voir Romilly si près de son berceau. $\|$ y vint avec son frère. C'était un plaisir de voir cet homme occupé de si grandes choses, si plein d'affection et de petits soins pour le frère, bon gros cultivateur qui, de son côté, ne cessait de le

par C. Navier, auquel Stéphane Flachat, partisan d'un canal «maritime » s'était opposé. M. I. Brunel qui connaissait Navier et les Flachat, a-t-il pour son propre projet discuté avec eux ?

166 É. Frère, « Notice historique... », op. cit., p. 93 « Appelé en France, en 1829, par le Gouvernement, pour donner les plans d'une distribution d'eau dans toutes les maisons de la capitale, à I'instar de ce qui existe à Londres, Brunel ne put s'entendre avec le Ministère Martignac. Certaines conditions que lui imposait ce ministère au sujet des machines et ustensiles à employer, parurent à Brunel un motif $d$ 'insuccès ... II ne fut pas plus heureux dans la proposition qu'il fit au Gouvernement français pour la création d'un chemin de fer de Paris à Rouen, par la vallée de la Seine, moyennant l'acceptation de capitaux anglais pour au moins un tiers de l'entreprise ». L'idée de confier à une compagnie privée la distribution d'eau à Paris, déjà présente en 1816, refit, en effet, surface en 1828. Des capitalistes anglais, les frères Lees, firent, entre autres des propositions. Un certain Lees ayant été en 1822 l'agent de M. I. Brunel à Paris, il est possible que ce soit de cette compagnie qu'il ait été l'agent en 1828. Laure BeaumontMaillet, L'eau à Paris, Paris, Hazan, 1991, p. 152.

167 Précis analytique des Travaux de l'Académie des Sciences, Belles lettres et Arts de Rouen, p. 5. "On voit au plaisir que prend $\mathrm{Mr}$ Brunel à entretenir ses compatriotes de ses travaux que s'ils sont exécutés en pays étranger, c'est à la France, au moins, qu'il voudrait pour ainsi dire faire la dédicace de la gloire qu'il en recueille... Afin de ne rien laisser désirer à I'Académie, M. Brunel a fait passer sous les yeux des membres de la Compagnie une perspective fort exacte de la galerie. Ce charmant diorama produit une complète illusion... ».

168 C'est sans doute à cette occasion que fut composé ce petit poème dont voici un extrait : «A. M. Brunel, auteur de la machine à faire des poulies et architecte du tunnel sous la Tamise-Air de la Pipe de Tabac ou bien air, Jeunes beautés au regard tendre: Brunel, grâce à la bienveillance / D'un frère ivre de tes succès / Nous pouvons (quelle jouissance !) / T'entendre et contempler tes traits / Que notre voix à ses oreilles / porte un remerciement flatteur : / S'il est beau de voir des merveilles, / II est bien doux d'en voir l'auteur ... Bientôt ta patrie adoptive / Dans son sein va te rappeler, / Et tous les cœurs de cette rive / Sur l'autre avec toi vont voler. / Qu'alors la Renommée en France / Vienne, et sensible à nos regrets, / Nous console de ton absence, / Par le récit de tes succès $»$. 
contempler avec un air d'admiration, mêlé de bonheur » . En 1831, un buste de lui en plâtre fut offert à la Société libre d'Émulation de Rouen dont il était correspondant par l'Abbé Gossier ${ }^{169}$. II revint à Hacqueville en $1834^{170}$. C'est de cette époque que datent les courriers adressés à plusieurs Normands dont son ami d'enfance Jacques Charles Allard, qui évoquent ses souvenirs de jeunesse et son séjour américain comme s'il était soucieux que la trace en soit conservée quelque part. L'ingénieur des Ponts-et-Chaussées, Pierre Frissard, alors en poste aux Andelys et qui deviendra l'année suivante ingénieur en chef du port du Havre, fit paraître cette même année à Évreux une notice sur le chantier du tunnel ${ }^{171}$. Après le décès de Marc Isambard en 1849, la presse nationale et surtout régionale lui consacra de nombreux articles. Une notice biographique parut dans les Mémoires de l'Académie de Rouen due à l'érudit normand Edouard Frère (1797-1874), ancien libraire, devenu archiviste de la Chambre de Commerce, qui visiblement le connaissait personnellement, de même du reste que le peintre et graveur, créateur du Musée des Antiquités de la ville, Hyacinthe Langlois (1777-1837) qui avait fait de lui un portrait gravé ${ }^{172}$. Une notice biographique parut encore en 1852 sous la plume de M. de Beaurepaire, ancien ministre plénipotentiaire, qui insista notamment sur ses entreprises avortées en France, vilipendant le manque de libéralisme français et l'omnipotence du Corps des Ponts-et-Chaussées ${ }^{173}$. Un monument fut enfin érigé

169 Séance publique de la Société libre d' Émulation de Rouen, 6 juin 1831.

170 R. Beamish, Memoir.., op. cit., p. 288, qui cite son journal : « Je n'ai rencontré que Pinchon, le menuisier de ma connaissance. Il ne me reconnut point lorsque je m'adressai à lui à la fenêtre. J'entrai par la fenêtre à son grand étonnement, et je lui dis qui j'étais. II me montra une partie du premier montant d'un octant que j'ai tenté, et enfin réussi à faire, ensuite quelques rouages ». 171 «Chemin souterrain sous la Tamise, dit tunnel », Bulletin de l'Académie Ebroïcienne, 1834.

172 « II avait été l'ami du savant Hyacinthe Langlois qui nous a laissé de lui un portrait gravé à l'eau forte », Journal de Rouen, 16 décembre $1849, n^{\circ} 350$. H. Langlois qui était l'illustrateur de la plupart des ouvrages d'Edouard Frère, avait aussi publié une petite notice sur M. I. Brunel ; Séance publique de la Société d'Emulation de Rouen, 1833, p. 133.

173 M. de Beaurepaire, Notice biographique de Marc-Isambard Brunel, Caen, 1852 (extrait de l'Annuaire normand). " Si M. Brunel appuyé de capitaux anglais qui se confiaient en lui pour obtenir un placement utile, avait pu, pour les chemins de fer, nous amener à entrer en 1829, dans la voie de confection intelligente et sage où il était un si bon conducteur, notre province aurait eu plus tôt le chemin de Paris à Rouen... ». L'article se terminait en faisant état d'un entretien qu'il aurait eu avec Isambard Kingdom : «En nous montrant la belle œuvre qu'il entreprenait alors (le tunnel), il reportait les yeux notre en 1854 à Hacqueville; il est toujours en place en face de sa maison natale et de l'église ${ }^{174}$. Le 25 septembre 1949, à l'instigation de son maire Auguste Belhoste, une cérémonie y fut organisée pour commémorer le centenaire de son décès ${ }^{175}$, à laquelle assista notamment un représen-tant de l'Académie des sciences dont il avait été élu membre correspondant en février $18288^{176}$.

Les choses se passèrent un peu différemment pour Isambard Kingdom. Sa renommée était devenue internationale et il conservait peu d'attaches avec la Normandie. Aussi l'écho fut-il surtout sensible dans la presse nationale. Flachat lui consacra une nécrologie dans le Bulletin de la Société des Ingénieurs civils, précédent un autre sur Robert Stephenson mort quelques mois après ${ }^{177}$. En 1864, parut aussi un article du Magasin Pittoresque étalé sur deux numéros décrivant la vie et les œuvres tant du père et du fils ${ }^{178}$. Mais c'est aussi déjà pour ses œuvres que Brunel fut honoré et suscita l'émerveillement, en particulier le Great Eastern dont le lancement défrayait la chronique au moment de son décès ${ }^{179}$. Un an après avoir fait l'objet d'une description enthousiaste dans l'ouvrage d'Eugène Flachat consacré aux navires transocéaniques,

patrie commune, et nous disait : On fait de pareils ouvrages dans ce pays, parce qu'on n'y a pas la Direction générale des pontset-chaussées ».

174 « À l'Angleterre, ses gigantesques travaux! À la France, à nous, son berceau et sa statue! », Le Vexin, 10 septembre 1854. 175 Le programme comportait l'inauguration d'une plaque commémorative sur sa maison natale, suivi d'un dépôt de gerbes à son monument, et après un vin d'honneur offert par le Conseil Municipal, un Grand bal de Nuit, avec l'orchestre "Scandaly Jazz » : Archives de l'Académie des sciences, dossier Brunel.

176 Dans la section Mécanique, sur la proposition de Charles Dupin. R. Beamish, Memoir.., op. cit., p. 270.

177 É. Flachat, Isambard Brunel, op. cit., p. 410 ; Robert Stephenson, op. cit., p. 418.

178 "Les deux ingénieurs Brunel », Le Magasin Pittoresque, trente-deuxième année, 1864, p. 138. La conclusion mérite d'être citée : "L'Angleterre, avide de gains au moins autant que de conquêtes, a reproché aux deux Brunel leur esprit aventureux. On disait du fils qu'il était le Napoléon des ingénieurs, pensant plus à la gloire qu'au profit, et plus à la victoire qu'aux dividendes. Cette tendance, à laquelle l'avait peut-être disposé son éducation en France, où l'honneur passe avant l'argent, nous paraît plus digne d'éloge que de blâme, surtout lorsqu'elle s'exerce dans la vaste et paisible sphère de l'industrie ».

179 Victor Hugo l'évoque dans La Légende des siècles (1859): " Le siècle a vu sur la Tamise / Croître un monstre, à qui l'eau sans borne fut promise, / Et qui longtemps, Babel des mers, eut Londres entier / Levant les yeux dans l'ombre au pied de son chantier / Effroyable, à sept mâts mêlant cinq cheminées / Qui hennissait au choc des vagues effrénées, / Emportant, dans le bruit des aquilons sifflant, / Dix mille hommes, fourmis éparses dans ses flancs, / Ce titan se rua, joyeux dans la tempête ; / Du dôme de Saint-Pierre son mât passait le faîte... ». 


\section{Les Brunel père et fils : deux célèbres ingénieurs anglais « Made in France "}

le Great Eastern fut acquis en 1867 par une société de droit français, la Société des affréteurs du Great Eastern $^{180}$, qui le fit rénover à grands frais à Liverpool afin qu'il puisse transporter les américains qui souhaitaient faire la traversée pour visiter l'Exposition Universelle. Jules Verne qui dès 1857 s'était juré de monter à bord de ce Léviathan, fut du voyage. II en tira un roman, Une Ville flottante ${ }^{181}$. On laissera le mot de la fin à Flachat

180 Sarl au capital de 2 millions F.

181 Jules Verne fit un rapide aller et retour, profitant de son court séjour en Amérique pour visiter les chutes du Niagara en remontant la rivière Mohawk. Environ 70 ans après lui et sans qui dès 1859 avait tenu ces propos : "L'esprit anglais 'ne fais que ce qui paie', est tout entier dans Stephenson. 'Il faut préparer l'avenir', répondait Brunel ... L'avenir n'a pas encore prononcé entre eux deux; le présent, avec une large part de bonheur, mais avec une bien plus grande part de jugement, a placé plus haut Stephenson dans I'histoire des entreprises financières... Au fond, cependant, l'incertitude existe encore... ». L'histoire a donné raison à Eugène Flachat. Isambard Kingdom Brunel occupe aujourd'hui la première place.

savoir sans doute, il fit ainsi le même parcours que M. I. Brunel, mais cette fois en train. 